EPJ manuscript No.

(will be inserted by the editor)

\title{
Conformational dynamics and internal friction in homo-polymer globules: equilibrium vs. non-equilibrium simulations
}

\author{
Thomas R. Einert ${ }^{1}$, Charles E. Sing ${ }^{2}$, Alfredo Alexander-Katz ${ }^{2}$, and Roland R. Netz ${ }^{3}$ \\ 1 Physik Department, Technische Universität München, James-Franck-Straße, 85748 Garching, Germany \\ 2 Department of Materials Science and Engineering, Massachusetts Institute of Technology, Cambridge, MA 02139-4307, U.S.A \\ ${ }^{3}$ Fachbereich Physik, Freie Universität Berlin, Arnimallee 14, 14195 Berlin, Germany, e-mail: rnetz@physik.fu-berlin.de
}

April 25, 2022

\begin{abstract}
We study the conformational dynamics within homo-polymer globules by solvent-implicit Brownian dynamics simulations. A strong dependence of the internal chain dynamics on the Lennard-Jones cohesion strength $\varepsilon$ and the globule size $N_{\mathrm{G}}$ is observed. We find two distinct dynamical regimes: a liquidlike regime (for $\varepsilon<\varepsilon_{\mathrm{s}}$ ) with fast internal dynamics and a solid-like regime (for $\varepsilon>\varepsilon_{\mathrm{s}}$ ) with slow internal dynamics. The cohesion strength $\varepsilon_{\mathrm{s}}$ of this freezing transition depends on $N_{\mathrm{G}}$. Equilibrium simulations, where we investigate the diffusional chain dynamics within the globule, are compared with non-equilibrium simulations, where we unfold the globule by pulling the chain ends with prescribed velocity (encompassing low enough velocities so that the linear-response, viscous regime is reached). From both simulation protocols we derive the internal viscosity within the globule. In the liquid-like regime the internal friction increases continuously with $\varepsilon$ and scales extensive in $N_{\mathrm{G}}$. This suggests an internal friction scenario where the entire chain (or an extensive fraction thereof) takes part in conformational reorganization of the globular structure.
\end{abstract}

\section{Introduction}

Conformational dynamics of polymers play a crucial role in biological systems. For example, pore-translocation of polymers such as RNA requires bond breaking and drastic conformational rearrangements to accommodate the geometrical constraints imposed by a pore [1-3]. Mechanical unfolding of biopolymers in force spectroscopy experiments induces profound conformational changes [4, which typically involve dissipative effects [5, 6. RNA sequences known as riboswitches experience conformational changes upon binding of small metabolites [7]. All of these transitions necessitate spatial rearrangements of the molecules and in certain cases require chain reptation within a collapsed region 8 . The time scale on which these changes happen is influenced by the medium, particularly the solvent viscosity, and by the polymer itself, through internal interactions. Therefore, if one desires to understand the dynamics of processes such as protein folding [9 12], packing of DNA in the chromosome [13-16], polymer collapse [17 19], or adsorption 20 22], all dissipation and viscous effects have to be considered 23.

While the viscosity of the medium certainly influences polymer chain dynamics, there are a number of effects that together give rise to what is called internal friction. Local interactions such as conformational transitions of backbone bonds and dihedral angles [9, 24, entanglement effects and excluded volume interactions in polymer systems, degrees of freedom orthogonal to the reaction coordinate [25, and the breakage and reformation of cohesive bonds [10, 26, 27. all lead to dissipation and thus increase the internal friction. For globular homopolymers, proteins in the molten globule phase 28, and disordered intermediates during protein folding [29, these effects can be conceptualized as roughness on a hypothetical free energy landscape, corresponding to many competing and intermediate states [30, 31, leading directly to the idea of an effective internal viscosity landscape 32 34. There have been a large number of coarse-grained simulation studies on the force-induced unfolding of proteins [35, globular polymers [17, 21, 36 38, and the diffusion of knots along a stretched chain 39 - 41 . Cohesive interactions between polymer monomers have been shown to lead to a phase transition from a liquid-like to a solid-like globule for long enough chains [42 50 .

In this paper we study internal friction in two model systems, which both can be realized experimentally. We perform solvent-implicit Brownian dynamics simulations on a homopolymer. Attractive interactions are modeled with a Lennard-Jones potential, where the cohesive strength $\varepsilon$ is varied. First, we study the diffusion of a globule, which forms from a polymer chain held at constant extension smaller than the contour length. This simulation is conducted under equilibrium conditions and no external forces are applied. For $\varepsilon<\varepsilon_{\mathrm{s}}$, where $\varepsilon_{\mathrm{s}}$ depends on the globule size, 
pronounced diffusion of the globule relative to the linker chain section is observed and characterized by the globule diffusivity $D_{\mathrm{G}} \cdot D_{\mathrm{G}}$ is a direct measure of the internal globule viscosity, since motion of the globule relative to the linker chain requires internal rearrangements. We observe that the diffusion constant is proportional to the reciprocal globule size $D_{\mathrm{G}} \sim N_{\mathrm{G}}^{-1}$ and shows a marked dependence on the cohesive strength $\varepsilon$. For $\varepsilon>\varepsilon_{\mathrm{s}}$, no diffusion is observed on the simulation time scales and the globule is stuck in a single conformation. This reflects the change of the internal dynamics going from liquid-like $\left(\varepsilon<\varepsilon_{\mathrm{s}}\right)$ to solid-like $\left(\varepsilon>\varepsilon_{\mathrm{s}}\right)$. Support for this interpretation is given by our second set of simulations, where we measure the dissipated work during stretching and unraveling the globule with a prescribed finite velocity. This is the same setup as studied by us before, but here with significantly longer chains and slower pulling velocities [32. Like in our equilibrium simulations, we observe two different regimes. For large $\varepsilon>\varepsilon_{\mathrm{s}}$, the force extension curves are characterized by pronounced fluctuations, which are absent for $\varepsilon<\varepsilon_{\mathrm{s}}$. Pulling decreases the number of monomers inside the globule, causing the fluctuations to vanish once $N_{\mathrm{G}}$ is below a certain threshold. Therefore, reducing the number of monomers $N_{\mathrm{G}}$ in the globule by stretching the chain, drives the system from the solid into the liquid state, similar to the reduction of the glass temperature of polymers close to interfaces 5154 . In the liquid state we perform extensive simulations and show that the internal viscosity is extensive, meaning that the dissipated work per monomer that is pulled out from the globule scales linearly with the globular monomer number $N_{\mathrm{G}}$ and the pulling velocity $v$, similar to our findings from the equilibrium simulations. For the chain dynamics this means that in the liquid-like regime, a model based on local viscous friction is valid, but that the viscosity depends on the size of a globule. Clearly, internal friction effects dramatically influences the time scales of chain dynamics in globules. In the solid-like regime, no definite conclusion on the conformational dynamics is possible from the simulations we performed.

\section{Brownian dynamics simulations}

We model the homopolymer by $N$ freely jointed beads of radius a, interacting with a potential $U\left(\left\{\boldsymbol{r}^{(N)}\right\}\right)$, which depends on the set of positions of all beads $\left\{\boldsymbol{r}^{(N)}\right\}$. The position $\boldsymbol{r}^{(i)}$ of the $i^{\text {th }}$ bead obeys the overdamped Langevin equation [55, 56]

$$
\frac{\partial \boldsymbol{r}^{(i)}}{\partial t}=-\mu_{0} \boldsymbol{\nabla}_{\boldsymbol{r}^{(i)}} U\left(\left\{\boldsymbol{r}^{(N)}\right\}\right)+\mu_{0} \boldsymbol{f}^{(i)}(t),
$$

where $\mu_{0}=1 /\left(6 \pi \eta_{0} a\right)$ is the Stokes mobility of a sphere with radius $a$ in a solvent with viscosity $\eta_{0} \cdot \boldsymbol{f}^{(i)}(t)$ is the random force acting on the $i^{\text {th }}$ bead. The components of the random force $f_{\alpha}^{(i)}(t), \alpha=x, y, z$, satisfy the StokesEinstein relation

$$
\left\langle f_{\alpha}^{(i)}(t) f_{\beta}^{(j)}\left(t^{\prime}\right)\right\rangle=\frac{2 \mathrm{k}_{\mathrm{B}} T}{\mu_{0}} \delta_{i j} \delta_{\alpha \beta} \delta\left(t-t^{\prime}\right)
$$

and are unbiased $\left\langle f_{\alpha}^{(i)}(t)\right\rangle=0$. Hydrodynamic interactions are neglected, since we are interested in the internal friction of the polymer caused by the monomer interactions. We express energies in units of the thermal energy $\mathrm{k}_{\mathrm{B}} T$, lengths in units of the bead radius $a$, and times in units of $\tau=a^{2} /\left(\mu_{0} \mathrm{k}_{\mathrm{B}} T\right)$, which is the characteristic diffusion time of a single bead. Using the dimensionless quantities $\tilde{t}=t / \tau, \tilde{\boldsymbol{r}}=\boldsymbol{r} / a, \tilde{\boldsymbol{f}}=\boldsymbol{f} /\left(\mathrm{k}_{\mathrm{B}} T / a\right)$, and $\tilde{U}=U /\left(\mathrm{k}_{\mathrm{B}} T\right)$, eq. (1) reads

$$
\frac{\partial \tilde{\boldsymbol{r}}^{(i)}}{\partial \tilde{t}}=-\nabla_{\tilde{\boldsymbol{r}}^{(i)}} \tilde{U}\left(\left\{\tilde{\boldsymbol{r}}^{(N)}\right\}\right)+\tilde{\boldsymbol{f}}^{(i)}(\tilde{t}) .
$$

For the Brownian dynamics simulation we use a discretized version of eq. (3) with a time step $\Delta t=0.0005 \tau$ and obtain the coordinates at time $t=n \Delta t[57$

$$
\tilde{\boldsymbol{r}}^{(i)}(n+1)=\tilde{\boldsymbol{r}}^{(i)}(n)+\left(-\nabla_{\tilde{\boldsymbol{r}}^{(i)}} \tilde{U}\left(\left\{\tilde{\boldsymbol{r}}^{(N)}\right\}\right)+\tilde{\boldsymbol{f}}^{(i)}(n)\right) \Delta \tilde{t}
$$

and

$$
\left\langle\tilde{f}_{\alpha}^{(i)}(n) \tilde{f}_{\beta}^{(j)}\left(n^{\prime}\right)\right\rangle=\frac{2}{\Delta \tilde{t}} \delta_{i j} \delta_{\alpha \beta} \delta_{n n^{\prime}}
$$

\section{Diffusivity of a globule along a periodic chain: equilibrium simulations}

In our preceding work we determined the internal viscosity $\eta_{\mathrm{G}}$ of a homopolymeric globule by measuring the dissipated energy when unfolding the globule by pulling apart the chain ends at finite speed [32]. We showed that for moderate 

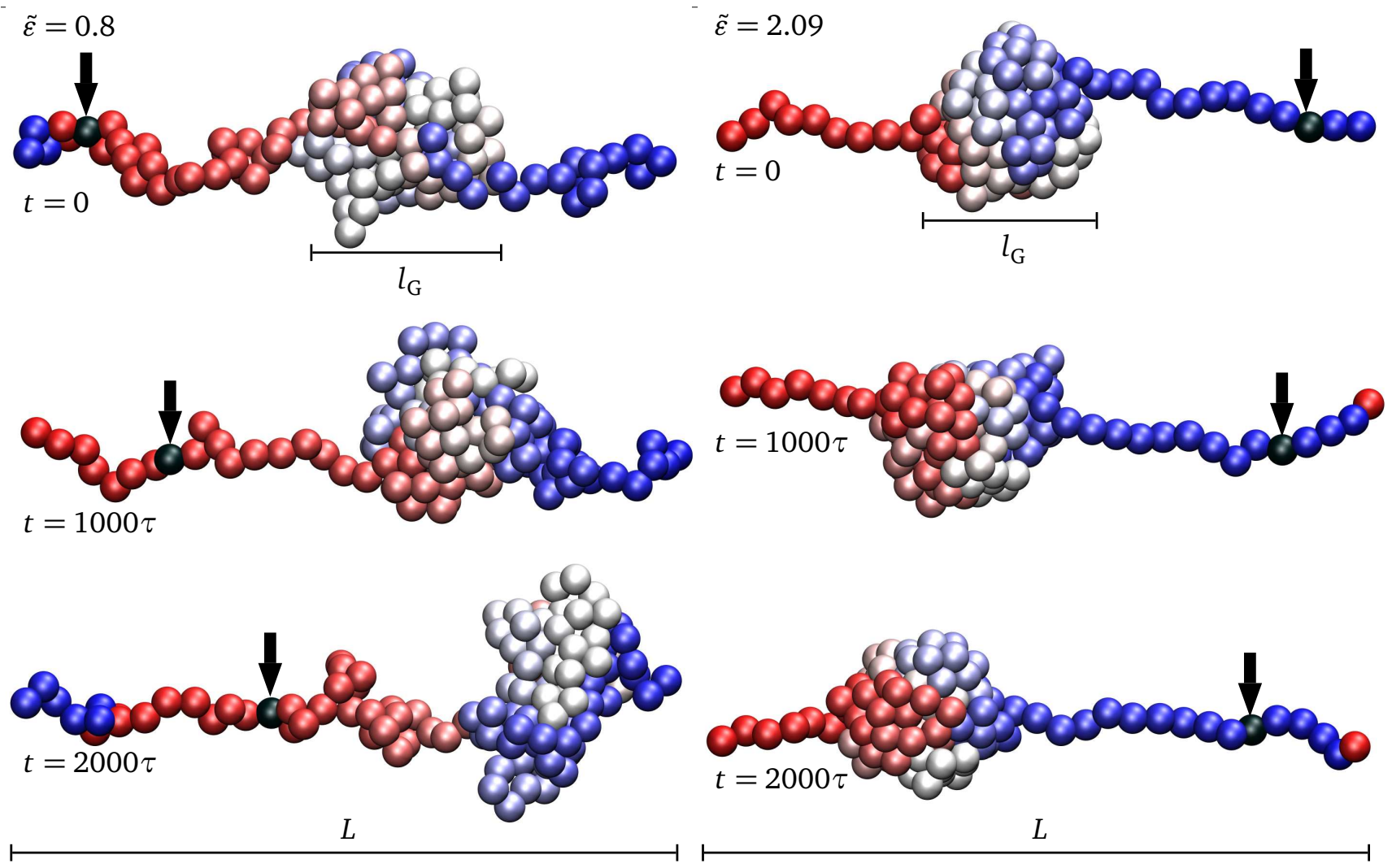

Figure 1: The diffusion of a globule relative to the stretched polymer linker is simulated. The globule mobility, the number of monomers $N_{\mathrm{G}}$ inside the globule, the diameter of the globule $l_{\mathrm{G}}$, and the fluctuations of the linkers (i.e. the stretched part of the chain that is not part of the globule) depend on the cohesive strength $\varepsilon$, eq. (10). Snapshots at different times for $\tilde{\varepsilon}=0.8$ (left) and 2.09 (right) are shown. To prevent motion of the linker chain section and thereby obtain directly the globule diffusivity relative to the chain, the monomer $i_{\mathrm{tr}}$ in the middle of the linker chain (indicated by the arrow) is trapped by a harmonic potential, eq. (11). Periodic boundary conditions for a box of length $L$ using the minimum image convention are employed to model an infinite polymer. The color coding indicates the running monomer index along the chain.

cohesion $\varepsilon$ the internal friction is the dominant dissipative effect as long as the majority of the monomers are part of the globule. However, as the globule is unraveled, the size of the globule decreases and more and more beads in the linker sections dissipate energy due to solvent friction. These effects had to be subtracted in order to obtain the internal globule friction. Likewise, reaching the relevant linear-response regime at low pulling velocities is subtle. Here, we introduce a novel system setup, where we perform equilibrium diffusion simulations with a globule of constant size: The entire polymer chain is held at constant extension smaller than the contour length, so that a globule forms for high enough cohesive energy. Since we fix the position of the linker chain in space, as we explain further below, the diffusive motion of the globule necessitates internal rearrangements, so that the globular diffusivity relative to the linker chain is a measure of the globule internal friction. Since the method works at equilibrium, the linear viscous regime is automatically obtained. By this method, the internal viscosity inside the globule manifests itself as a macroscopic and experimentally observable quantity: the diffusivity of the globule, $D_{\mathrm{G}} \sim 1 / \eta_{\mathrm{G}}$. This system could be realized experimentally by a polymer held at constant extension in an optical or magnetic tweezers setup 58. In such an experiment the cohesive force can be varied by changing the solution conditions, whereas the size of the globule can be varied by changing the trap distance.

\subsection{Model}

\subsubsection{Description of the system}

We consider a polymer held at a fixed extension $L=50 a$ in the $x$-direction, which is smaller than the contour length $L_{\mathrm{c}}=2 a(N-1)$ of the polymer. A globule will form for large enough attractive Lennard-Jones interaction between the 
monomers, see fig. 1. To eliminate finite size effects, we introduce periodic boundary conditions in $x$-direction, which are implemented via the minimum image convention [59]: The components of the vector pointing from $\boldsymbol{r}^{(i)}$ to $\boldsymbol{r}^{(j)}$ are given by

$$
\begin{aligned}
& r_{x}^{(i, j)}=\mathcal{D}\left(r_{x}^{(i)}, r_{x}^{(j)} ; L\right) \equiv\left(\left(r_{x}^{(j)}-r_{x}^{(i)}+3 L / 2\right) \bmod L\right)-L / 2 \\
& r_{y / z}^{(i, j)}=r_{y / z}^{(j)}-r_{y / z}^{(i)} .
\end{aligned}
$$

We use a box size $L=50 a$ in all simulations. The potential energy has four contributions

$$
U=U_{\mathrm{b}}+U_{\mathrm{r}}+U_{\mathrm{LJ}}+U_{\mathrm{tr}} .
$$

$U_{\mathrm{b}}$ and $U_{\mathrm{r}}$ are the bond potentials acting between neighboring monomers. The backbone bonds are modeled by harmonic potentials

$$
U_{\mathrm{b}}=\frac{\kappa}{2} \sum_{i=1}^{N-1}\left(r^{(i, i+1)}-2 a\right)^{2},
$$

with $\kappa=200 \mathrm{k}_{\mathrm{B}} T / a^{2}$ and $r^{(i, j)}=\left|\mathbf{r}^{(i, j)}\right|$, see eq. (6). As periodic boundary conditions are employed, the polymer forms a closed ring, which is achieved by connecting the first and last monomer by

$$
U_{\mathrm{r}}=\frac{\kappa}{2}\left(r^{(N, 1)}-2 a\right)^{2} \text {. }
$$

The monomer cohesion and excluded volume interactions are modeled with a Lennard-Jones potential

$$
U_{\mathrm{LJ}}=\varepsilon \sum_{i=1}^{N} \sum_{j=1}^{i-1}\left(\left(\frac{2 a}{r^{(i, j)}}\right)^{12}-2\left(\frac{2 a}{r^{(i, j)}}\right)^{6}\right) .
$$

$\varepsilon=0$ models an ideal phantom chain without excluded volume interactions and attractive interactions between the monomers. For $\varepsilon>0$ the first term in eq. 100 accounts for the repulsive excluded volume interaction at short separations, whereas the second term is responsible for cohesion which reflects hydrophobic attraction between monomers in a solvent-implicit fashion. For $0 \leq \varepsilon<\varepsilon_{\mathrm{G}} \approx 0.5$ the polymer is in the swollen state and no globule exists. Increasing $\varepsilon$ above $\varepsilon_{\mathrm{G}}$ causes the polymer to collapse and a globule forms. The static globule behavior from our simulations agrees with previous work [17, 32, 42, 50, 60. The value of the cohesive strength at the globule transition, $\varepsilon_{\mathrm{G}}$, depends on the system size [43]. For even larger values $\varepsilon>\varepsilon_{\mathrm{s}}$ a solid phase appears.

As we are interested in the relative motion of the globule with respect to the rest of the chain, we prevent the linker chain from moving by an external trapping potential acting on one monomer, $U_{\mathrm{tr}}$, which can be viewed as the effect of an e. g. optical tweezers. The linker chain is the stretched part that does not belong to the globule, fig. 1. We introduce a harmonic trap potential

$$
U_{\mathrm{tr}}=\frac{\kappa_{\mathrm{tr}}}{2}\left(\boldsymbol{r}^{\left(i_{\mathrm{tr}}\right)}-\boldsymbol{R}\right)^{2},
$$

which is located at $\boldsymbol{R}$ and acts on bead $i_{\mathrm{tr}}$ that is in the middle of the linker, with stiffness $\kappa_{\mathrm{tr}}=10 \mathrm{k}_{\mathrm{B}} T / a^{2}$. The index of the trapped bead $i_{\mathrm{tr}}$ depends on the position of the globule and on the set of beads that belong to the globule. The exact definition of the globule is described in the next section. Due to globule motion, the index of the bead in the middle of the linker might change from $i_{\operatorname{tr}}$ at time $t$ to $i_{\mathrm{tr}}^{\prime}$ at some later time $t^{\prime}$. If this happens, we update the $x$-coordinate of the trap position to the new position $R_{x}\left(t^{\prime}\right)=R_{x}(t)+2 a \mathcal{D}\left(i_{\mathrm{tr}}, i_{\mathrm{tr}}^{\prime} ; N\right)$, eq. $[6]$, and the trapping force then acts on bead $i_{\mathrm{tr}}^{\prime}$. The trapped beads in the snapshots shown in fig. 1 are indicated by arrows.

This setup allows us to study the diffusion of the globule relative to the linker chain along the $x$-axis. The effect of using periodic boundary conditions rather than long linkers to each side is fourfold. First, the simulation is sped up as the system is smaller. Second, the globule is stabilized as the configurational space for the unfolded system is reduced since only fluctuations up to a wavelength of the order of the simulation box are possible. Third, motion of the globule as a whole without internal friction is reduced. Fourth, knots may not form as the polymer forms a closed ring.

\subsubsection{Definition of the globule}

We partition the $x$-axis into bins of width $l_{\mathrm{b}}=2 a$ and measure the monomer density $\rho_{k}$ in the $k^{\text {th }}$ bin, $k=1, \ldots, L / l_{\mathrm{b}}$. The globule is defined as the region, where the monomer density projected on the $x$-axis fulfills the condition $\rho_{k}>5 / a$. 


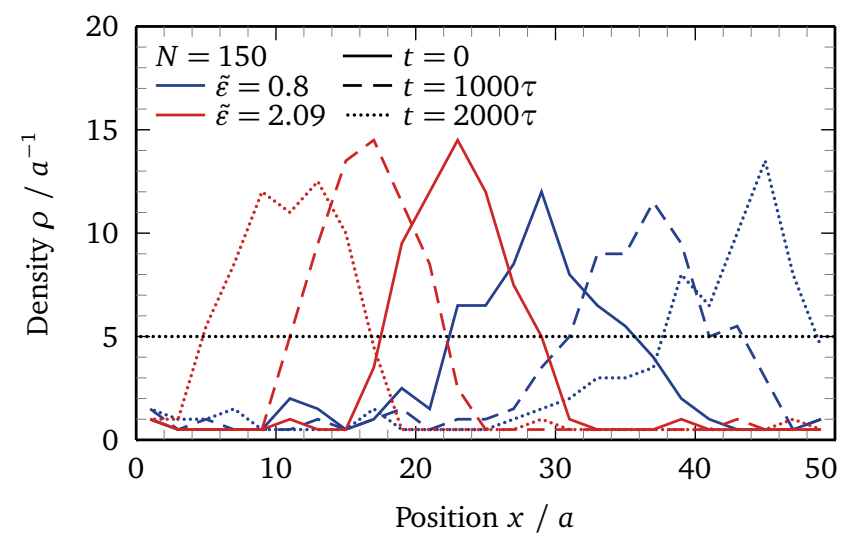

Figure 2: The density profile of the globule depends on the cohesive strength $\varepsilon$. The globule is defined as the region where the monomer density is $\rho>5 / a$ (indicated by the horizontal line).

In fig. 2 the density profiles of the snapshots in fig. 1 are shown. If at the edge of the globule the density profile is not monotonous and $\rho_{k}<5 / a$, but $\rho_{k-1}, \rho_{k+1}>5 / a$, we account for such cases by adding these bins to the globule, too. This ensures that we end up with a list of bins that are connected. For small $\tilde{\varepsilon}<1$ and small $N<150$ it is possible that - according to the above definition - more than one globule exists or that the globule is smeared out over the complete simulation box, however we will not consider simulations in which this occurs. Such complications are never observed for $\tilde{\varepsilon}>1$ and $N>150$.

\subsubsection{Definition of the index of the trapped bead}

Let us first define the index of the bead $i_{\mathrm{r}}$, which is at the right edge of the globule, and the index of the bead $i_{1}$, which is at the left edge of the globule. $i_{\mathrm{r}}$ is obtained by picking a monomer inside the globule and moving along the chain contour with increasing monomer index. $i_{\mathrm{r}}$ is the largest index that is still in a bin belonging to the globule. We also check for loops, which leave the globule and return again: If a loop occurs, we add all monomers of the loop to the globule even if they lie in a bin outside the globule. $i_{1}$ is defined in the same way yet by decreasing the index. The index of the central bead $i_{\mathrm{c}}$ in the middle of the globule and the number of monomers $N_{\mathrm{G}}$ inside the globule are defined as $i_{\mathrm{c}}=\left(i_{1}+i_{\mathrm{r}}\right) / 2, N_{\mathrm{G}}=i_{\mathrm{r}}-i_{1}+1$ if $i_{1}<i_{\mathrm{r}}$ and $i_{\mathrm{c}}=\left(i_{1}-N+i_{\mathrm{r}}\right) / 2, N_{\mathrm{G}}=i_{1}-i_{\mathrm{r}}+1$ if $i_{\mathrm{l}}>i_{\mathrm{r}}$. The index of the central bead $i_{\mathrm{c}}$ yields the index of the trapped bead via $i_{\mathrm{tr}}=i_{\mathrm{c}}+N / 2$, which therefore depends on the motion of the globule. We could have also trapped a fixed monomer for the whole duration of the simulation. Our procedure (i.e. trapping a bead that by construction is never part of the globule) allows maximal dynamic freedom for the globule and thus improves equilibration of the system.

\subsection{Results}

\subsubsection{Number of monomers inside the globule}

The number of monomers $N_{\mathrm{G}}$ inside the globule depends on the chain length $N$ and on the cohesive strength $\varepsilon$, fig. 3 a. Increasing $\varepsilon$ raises $N_{\mathrm{G}}$ only up to a limiting value $N_{\mathrm{G}}^{*}$, which is determined by the equation

$$
\left(N_{\mathrm{G}}^{*}\right)^{1 / 3}+\left(N-N_{\mathrm{G}}^{*}\right)=L /(2 a) .
$$

Eq. 12 describes a close-packed spherical globule consisting of $N_{\mathrm{G}}^{*}$ monomers with a tightly stretched linker consisting of $N-N_{\mathrm{G}}^{*}$ monomers. As the limiting value of our simulations coincides with the predicted value of eq. (12), as shown in fig. 3a , our definition of the globule is justified. For large $\varepsilon$, the linkers are completely stretched, as the energetic gain of a monomer joining the globule outweighs the entropic loss of reducing the fluctuations of the linker. For small $\varepsilon$, monomers are not tightly bound to the globule as can be seen qualitatively from fig. 1 . Therefore, size fluctuations of the globule are more substantial for small cohesive strengths and decrease upon increasing $\varepsilon$, as shown in fig. $3 \mathrm{p}$. 


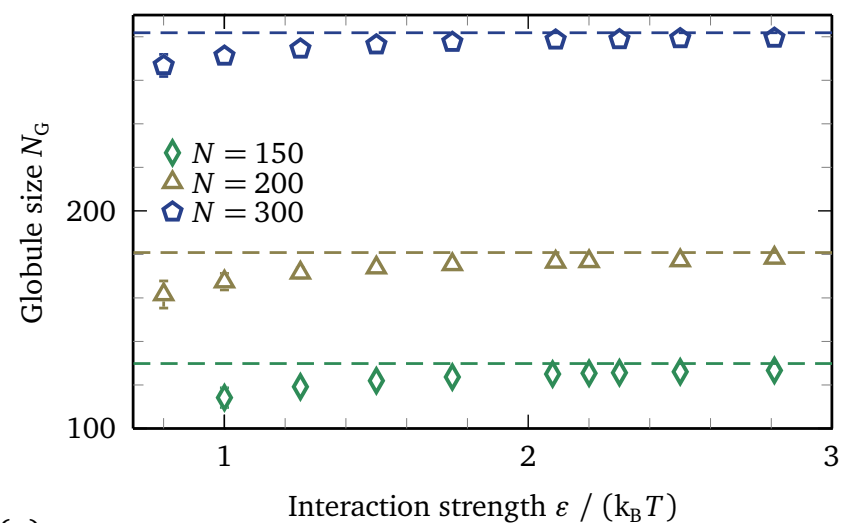

(a)

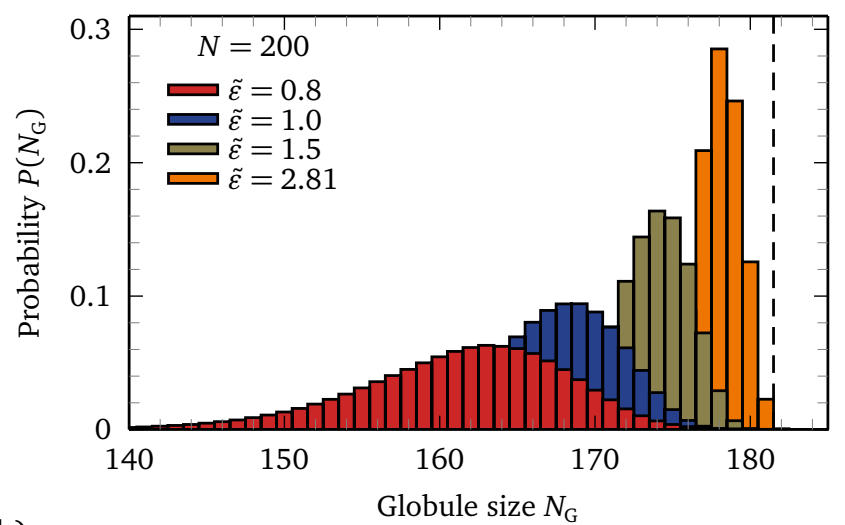

(b)

Figure 3: (a) The number of monomers $N_{\mathrm{G}}$ inside the globule increases weakly with the interaction strength $\varepsilon$ until it finally levels off. The limiting values $N_{\mathrm{G}}^{*}$ are depicted by horizontal broken lines and are determined by eq. (12), describing a spherical globule and tightly stretched linkers. Error bars denote the standard deviations of the distributions. (b) Probability distribution of the number of monomers $N_{\mathrm{G}}$ inside the globule for chain length $N=200$ and different cohesive strengths $\tilde{\varepsilon}=0.8,1,1.5,2.81$. For small $\varepsilon$ considerable size fluctuations are observed, which decrease upon increasing $\varepsilon$. Further, increasing $\varepsilon$ raises the mean $N_{\mathrm{G}}$ up to the limiting value $N_{\mathrm{G}}^{*}$ given by eq. 12 (broken vertical line).

\subsubsection{Definition of the center of the globule}

In order to properly describe the motion of the polymer in a simulation box with periodic boundary conditions in $x$-direction, we introduce non-periodic polymer coordinates $\left\{\hat{\boldsymbol{r}}^{(N)}\right\}$, which are not restricted to the primary simulation cell. They are defined recursively starting from the first monomer with $\hat{\boldsymbol{r}}^{(1)}=\boldsymbol{r}^{(1)}$ via

$$
\hat{\boldsymbol{r}}^{(i+1)}=\hat{\boldsymbol{r}}^{(i)}+\boldsymbol{r}^{(i, i+1)}
$$

for $i>1$, where $\boldsymbol{r}^{(i, i+1)}$ is given by eq. (6). Since we are considering a ring-like polymer with periodic boundary conditions in the $x$-direction, the center of mass of the globule has to be defined carefully. The center of the globule $\boldsymbol{r}^{(\mathrm{G})}$ is calculated by using the non-periodic polymer coordinates, eq. (13), according to

$$
\boldsymbol{r}^{(\mathrm{G})}=\frac{1}{N_{\mathrm{G}}} \sum_{i=i_{1}}^{i_{\mathrm{r}}} \hat{\boldsymbol{r}}^{(i)}
$$

Periodic boundary conditions may introduce jumps of the size of the box $L$ in the trajectory $\boldsymbol{r}^{(\mathrm{G})}(t)$. We remove those jumps by connecting the value of any quantity $q(t)$ at time $t$, which is subject to periodic boundary conditions, with the value $q(t-\Delta t)$ in the previous time step via the minimum image condition.

\subsubsection{Diffusivity of the globule}

Our setup enables us to study the motion of the globule in space in a fashion that is coupled to its internal conformational dynamics. In other word, since we fix the linker chain in space, only internal chain rearrangements within the globule lead to diffusion of the globule in space. In fig. 4 trajectories of the $x$-coordinate $r_{x}^{(\mathrm{G})}$ of the center of the globule, eq. (14), are shown for $N=200$ and various cohesive strengths $\varepsilon$. Fig. 4 demonstrates that the diffusivity decreases with increasing $\varepsilon$. As the monomers become more cohesive, it is more difficult for the globule to rearrange internally and hence to move. This effect is further accented by the increase in globule size $N_{\mathrm{G}}$, see fig. 3 , which additionally decreases the mobility of the globule.

To quantify these observations, we calculate the mean squared displacement (MSD) of $r_{x}^{(\mathrm{G})}$. For normal diffusive behavior one expects the MSD to scale linearly with time and to be characterized by the diffusion constant $D_{\mathrm{G}}$

$$
\operatorname{MSD}(t)=\left\langle\left(r_{x}^{(\mathrm{G})}(t)-r_{x}^{(\mathrm{G})}(0)\right)^{2}\right\rangle=\frac{1}{T} \int_{0}^{T}\left(r_{x}^{(\mathrm{G})}\left(t+t^{\prime}\right)-r_{x}^{(\mathrm{G})}\left(t^{\prime}\right)\right)^{2} \mathrm{~d} t^{\prime}=2 D_{\mathrm{G}} t
$$




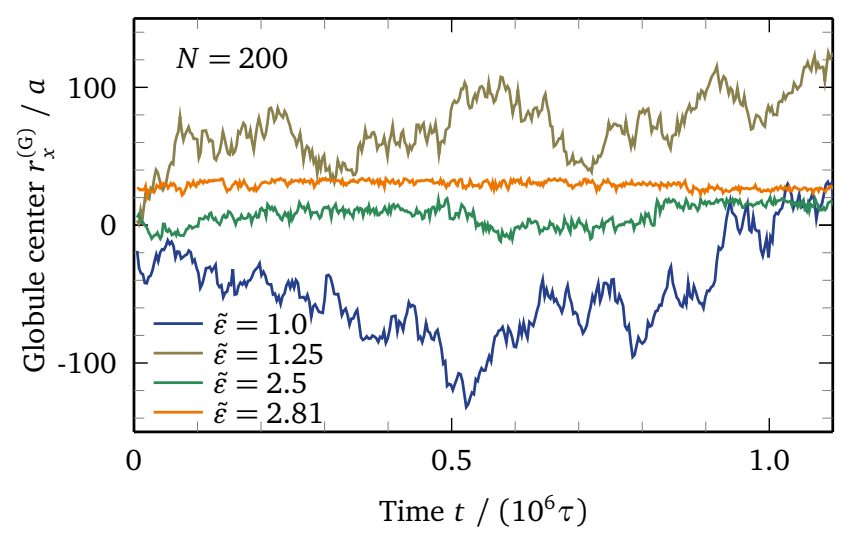

Figure 4: Trajectories of the $x$-coordinate of the center of the globule, $r_{x}^{(\mathrm{G})}$, defined in eq. (14), for $N=200$ and various interaction strengths $\tilde{\varepsilon}=1,1.25,2.5,2.81$. The smaller the attractive interaction between the monomers, the more mobile the globule is. If $\varepsilon$ is large, the globule is frozen in a single conformation and does not move at all.

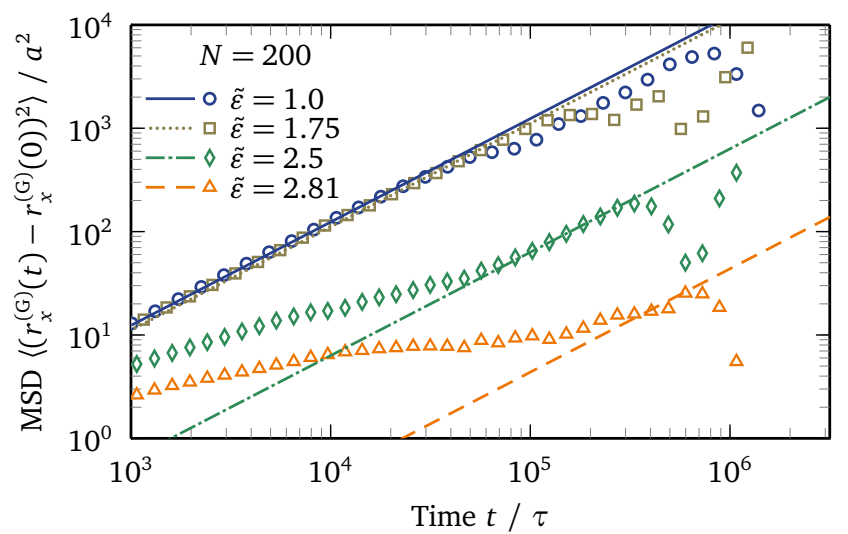

Figure 5: The mean squared displacement (MSD) of the center of the globule for $N=200$ and $\tilde{\varepsilon}=1,1.75,2.5,2.81$ is calculated from the trajectories, fig. 4, using eq. (15). Symbols denote the measured MSD from our simulations, lines the corresponding linear fits for $\operatorname{MSD}(t) \gtrsim 10$. For $\tilde{\varepsilon}<\tilde{\varepsilon}_{\mathrm{s}}$ with $\tilde{\varepsilon}_{\mathrm{s}} \approx 2.3$ for $N=200$, the globule exhibits normal diffusion and the diffusivity $D_{\mathrm{G}}$ decreases as $\varepsilon$ increases. For $\tilde{\varepsilon}=2.5$, normal diffusive behavior is obtained, whereas for $\tilde{\varepsilon}=2.81$ the diffusion time scale is of the order of the simulation time and normal diffusive behavior is barely reached.

MSD curves for $N=200$ and various cohesive strengths are shown in fig. 5 on a double logarithmic plot. The MSD curves are fitted with linear functions for $\operatorname{MSD}(t) \gtrsim 10$ in order to obtain the diffusion constant $D_{\mathrm{G}}$. For small $\varepsilon<\varepsilon_{\mathrm{s}}$, normal diffusion is observed with $D_{\mathrm{G}}$ decreasing as $\varepsilon$ increases. However, as can be seen in fig. 5 for $N=200$ and $\tilde{\varepsilon}=2.5$, the normal diffusive regime only occurs at very long time scales and for $\tilde{\varepsilon}=2.81$ is barely reached on the time scales of our simulations. We attribute this to a change of the internal dynamics of the globule, which is slowed down with increasing cohesion and is effectively suppressed for $\varepsilon>\varepsilon_{\mathrm{s}}$. One remaining pathway for the globule to rearrange at very large cohesion is to dissolve - at least partly - and refold into a different configuration. As a consequence, the time scale characterizing the internal dynamics should become comparable to the time scale on which the globule dissolves. This dissolution time is huge as it scales exponentially with $\varepsilon N_{\mathrm{G}}$ and is beyond our simulation time. For that reason we observe only stuck globules for large cohesive strengths, which remain in a single conformation. The fitted diffusion constants are shown in fig. 6 and contrasted with an idealized limit, where internal friction is absent and the globule and the linker move independently. The diffusivity in this limit is given by the Rouse diffusion constant of $N_{\mathrm{G}}^{\prime}$ monomers 55 .

$$
D_{0}=\frac{\mu_{0} \mathrm{k}_{\mathrm{B}} T}{N_{\mathrm{G}}^{\prime}} .
$$




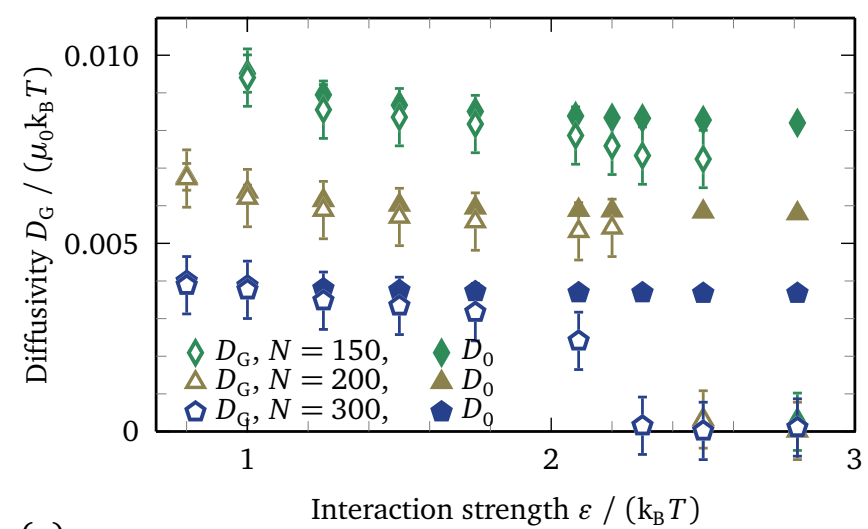

(a)

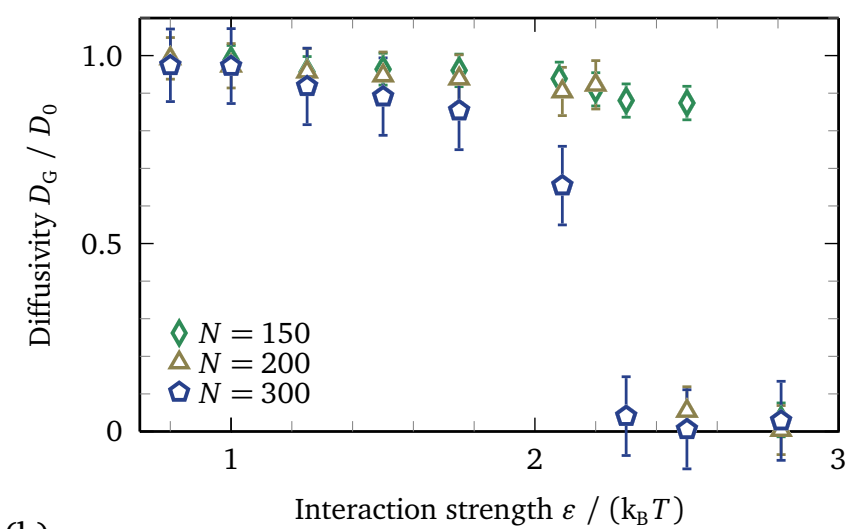

(b)

Figure 6: (a) Diffusion constant $D_{\mathrm{G}}$ of the globule (open symbols) as obtained from linear fits to the MSD curves, fig. 5 $D_{\mathrm{G}}$ decreases with increasing $N$ and $\varepsilon$. At $\varepsilon=\varepsilon_{\mathrm{s}}$, with $\varepsilon_{\mathrm{s}}$ depending on $N$, a transition of the internal dynamics occurs and the diffusion constant drops to zero. $D_{\mathrm{G}}$ is compared to the ideal Rouse diffusion constant $D_{0}$ (solid symbols) of a globule with $N_{\mathrm{G}}^{\prime}$ monomers, eqs. (16) and (17), which can move freely and independent of the linkers. (b) Rescaling the actual diffusion constant by the ideal diffusion constant, $D_{\mathrm{G}} / D_{0}$, removes the $N$ dependence to a large extent in the liquid regime.

$N_{\mathrm{G}}^{\prime} \leq N_{\mathrm{G}}$ is the reduced number of monomers within the globule that actually have to comove when the globule is displaced by some distance $x . N_{\mathrm{G}}^{\prime}$ can be estimated by continuing the linker through the globule and subtracting the number of monomers, which belong to this internal linker section, from $N_{\mathrm{G}}$, see fig. 7 for an illustration,

$$
N_{\mathrm{G}}^{\prime}=N_{\mathrm{G}}-\left(N-N_{\mathrm{G}}\right) \frac{l_{\mathrm{G}}}{L-l_{\mathrm{G}}} .
$$

In fig. 6 a pronounced dependence of the diffusion constant $D_{\mathrm{G}}$ (open symbols) on the globule size $N_{\mathrm{G}}$ is observed. The diffusivity decreases as the internal interactions $\varepsilon$ increase. This is due to the coupling of the internal dynamics of the globule to the overall motion of the globule, because, as explained before, in our simulation setup the linker is fixed and the globule can only move via internal conformational chain reorganization. Slowing down the internal dynamics by increasing $\varepsilon$ thus reduces the mobility of the globule. For small $\varepsilon$ one observes $D_{\mathrm{G}} \approx D_{0}$ (filled symbols), implying that internal friction is unimportant. Conversely, increasing $\varepsilon$ causes increasing deviations between the mobility of the globule and the ideal system, until finally $D_{\mathrm{G}}$ drops to zero as the liquid-solid transition is crossed. For $\varepsilon>\varepsilon_{\mathrm{s}}$ the globule is in a frozen state with strongly suppressed internal dynamics. Since the linkers are trapped, this also impedes the motion of the globule as a whole and leads to a vanishing diffusion constant. To disentangle size from cohesive effects, we show the rescaled diffusivity $D_{\mathrm{G}} / D_{0}$ in fig. $6 \mathrm{~b}$. For $\varepsilon \rightarrow 0$ the rescaled diffusivity approaches unity, indicating that internal friction is unimportant. The rescaled diffusivity exhibits for small cohesion only a weak dependence on the size of the globule. As $\varepsilon$ approaches the solid regime, however, deviations between different system sizes become observable. Thus, we propose that in the liquid regime, the internal friction is extensive and to leading order scales with the size of the globule

$$
D_{\mathrm{G}} \sim 1 / N_{\mathrm{G}}^{\prime}
$$

as will be corroborated by our non-equilibrium simulations in section 4 . Further below we will also show how the internal viscosity within the globule can be extracted from the diffusivity ratio $D_{\mathrm{G}} / D_{0}$.

\section{Forced unfolding of globules: non-equilibrium simulations}

\subsection{Model}

We now discuss our simulations for the non-equilibrium force-induced dissolution of homopolymeric globules. Here, the two chain ends are positionally constraint at $\boldsymbol{r}^{(1)}(t)=-\boldsymbol{R}(t)$ and $\boldsymbol{r}^{(N)}(t)=\boldsymbol{R}(t)$. The trap positions are moved at constant speed $R_{x}(t)=R_{x}^{(\min )}+v t$ with $R_{y / z}=0$, from $R_{x}^{(\min )}$ up to a maximal position $R_{x}^{(\max )}=a(N-1)$, producing a time-varying chain extension $x(t)=2 R_{x}(t)$. During the pulling, the force acting on the terminal beads is measured. The backbone bonds are modeled by a harmonic potential $U_{\mathrm{b}}$ as before, see eq. (8). Excluded volume 


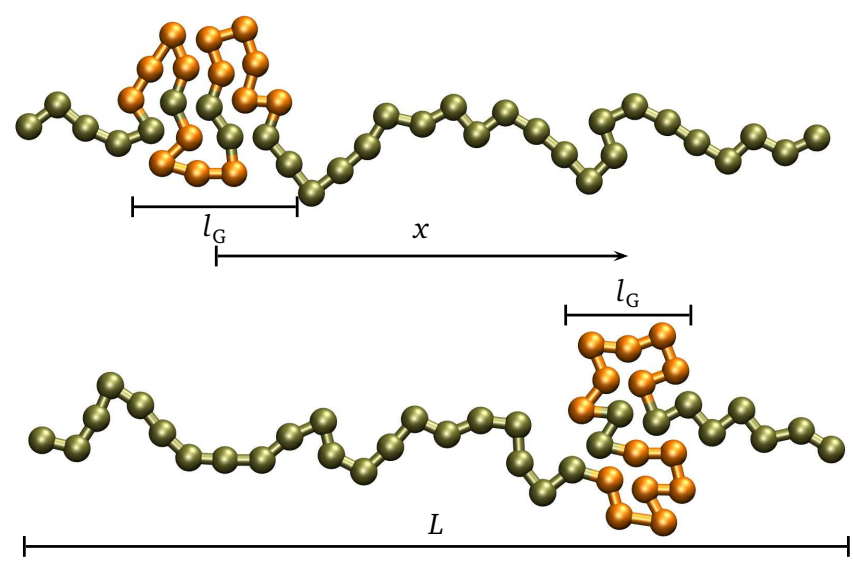

Figure 7: Illustration of the concept and calculation of the reduced number of monomers $N_{\mathrm{G}}^{\prime}$ in the globule. For a displacement of the globule by a distance $x$ only a reduced number of monomers have to move along, here $N_{\mathrm{G}}^{\prime}=14$ (shown in orange). In order to obtain $N_{\mathrm{G}}^{\prime}$, the linker is continued through the globule and the monomers belonging to this internal linker are subtracted from $N_{\mathrm{G}}$ yielding $N_{\mathrm{G}}^{\prime}$, see eq. (17).

and cohesive interactions are again modeled by a Lennard-Jones potential $U_{\mathrm{LJ}}$, eq. (10). Eq. (4) is used to integrate the Langevin equation. We no longer employ periodic boundary conditions, however still prevent knot formation by introducing a potential, which mimics two repulsive bars that extend from the first/last bead to the left/right along the $x$-axis

$$
U_{\mathrm{k}}= \begin{cases}\sum_{i=2}^{N-1}\left(\left(2 a / \hat{\rho}^{(i)}\right)^{12}-2\left(2 a / \hat{\rho}^{(i)}\right)^{6}+1\right) & \text { if } \hat{\rho}^{(i)}<2 a \text { and }\left|r_{x}^{(i)}\right|>R_{x} \\ 0 & \text { else, }\end{cases}
$$

$\hat{\rho}^{(i)}=\sqrt{r_{y}^{(i)^{2}}+r_{z}^{(i)^{2}}}$. $U_{\mathrm{k}}$ does not affect the stretching response. The total energy is therefore given by $U=$ $U_{\mathrm{b}}+U_{\mathrm{LJ}}+U_{\mathrm{k}}$. Two different protocols, annealed and un-annealed, are used for the initial configurations in order to investigate the history dependence on the globule pulling response. We record force extension curves for various chain lengths $N=50,100,200,300$, cohesive strengths $0 \leq \tilde{\varepsilon} \leq 4.1$, and pulling velocities $\tilde{v}=v /(a / \tau)=$ $0.001,0.0045,0.01,0.0225,0.045$ going significantly beyond our previous work, where the largest system was $N=100$, the slowest velocity was $\tilde{v}=0.0045$, and only un-annealed initial configurations were used [32]. For each parameter set, twenty stretching cycles are simulated.

\subsubsection{Annealed initial structures}

Annealed initial structures are obtained by performing an equilibrium annealing simulation with moderate cohesive strength $\tilde{\varepsilon}=0.8$ and fixed trap position $R_{x}=0.1 a N$. The LJ interaction is strong enough to induce globule formation, yet small enough to allow for rapid equilibration of the chain conformation inside the weakly collapsed globule. Knot formation is prevented by virtue of the potential in eq. 119 . Every $t=20000 \tau$ a structure is recorded, which is subsequently equilibrated for $t=10000 \tau$ using the target cohesive strength at which the pulling simulation is to be conducted. The resulting structure is used as one initial configuration for the subsequent pulling cycle. In fig. $8 \mathrm{a}$ the pulling protocol is illustrated and in fig. 9 typical initial configurations are depicted.

\subsubsection{Un-annealed initial structures}

This set of initial structures is obtained by starting from an extended configuration and moving the traps from $R_{x}^{(\max )}=a(N-1)$ to $R_{x}=0.03 a N$. Typically, we observe the formation of one globule for $\tilde{v}<0.01$ and the formation of two globules near the traps for $\tilde{v}>0.01$, which merge at small extension, see snapshots in fig. 8 $\mathrm{b}$. Without pausing, the traps are subsequently extended to $R_{x}=0.1 a N$, where the actual pulling cycle starts and force extension curves are recorded for further analysis. During the compression stage, the traps are moved with the same velocity $v$ with which the force extension curve is recorded, fig. $8 \mathrm{p}$. For larger cohesive strengths $\varepsilon$, non-equilibrium ordered structures prevail as initial configurations for the subsequent pulling cycle, see fig. 9 for an illustration. Although not the prime target of our present investigation, such structures might be of relevance when studying the dynamics and packing of DNA chromatin structures far from equilibrium [13. 15. 

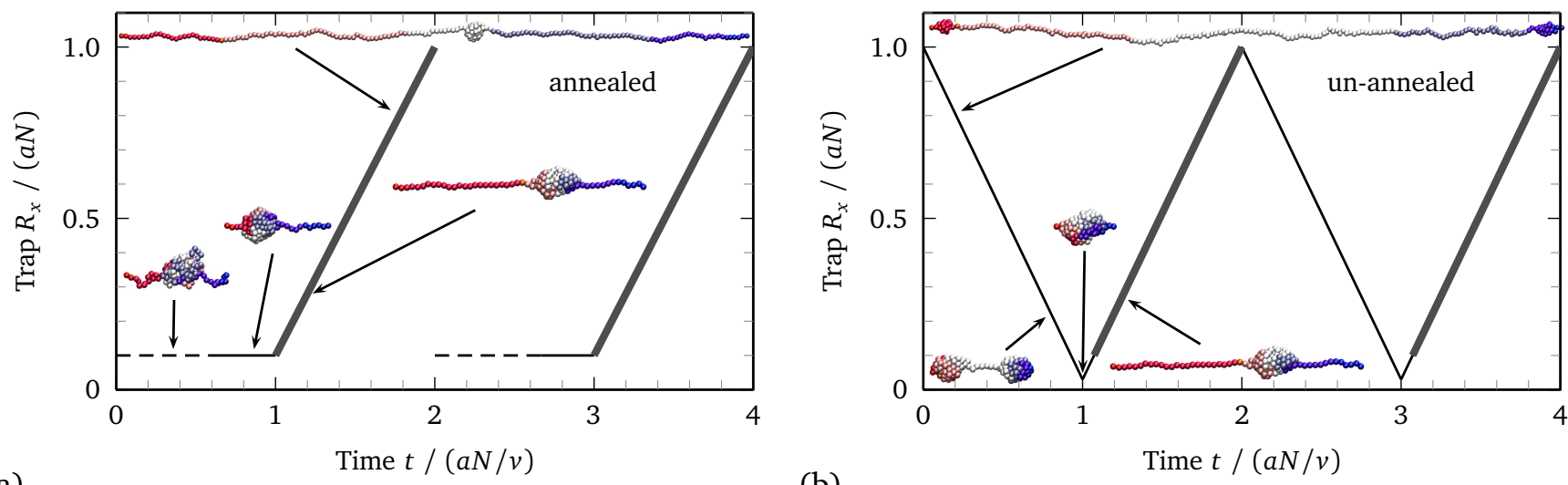

(a)

Figure 8: Illustration of the two different pulling protocols. $R_{x}$ and $-R_{x}$ denote the positions of the two traps that positionally constrain the chain ends. (a) Preparation of the annealed structures. After a long equilibrium run with $\tilde{\varepsilon}=0.8$ and $R_{x}=0.1$ (broken line), the globule is equilibrated at the cohesive strength at which the pulling curve is recorded (horizontal solid line). The subsequent pulling cycle is depicted by the thick gray line. (b) Preparation of the un-annealed structures. The traps are moved from complete extension, $R_{x}=a(N-1)$, to $R_{x}=0.03 a N$, which ensures that one single globule forms. Without pausing, the pulling cycle starts and force extension curves are recorded and analyzed in the interval $0.1<R_{x} /(a N)<1$ (thick gray line).

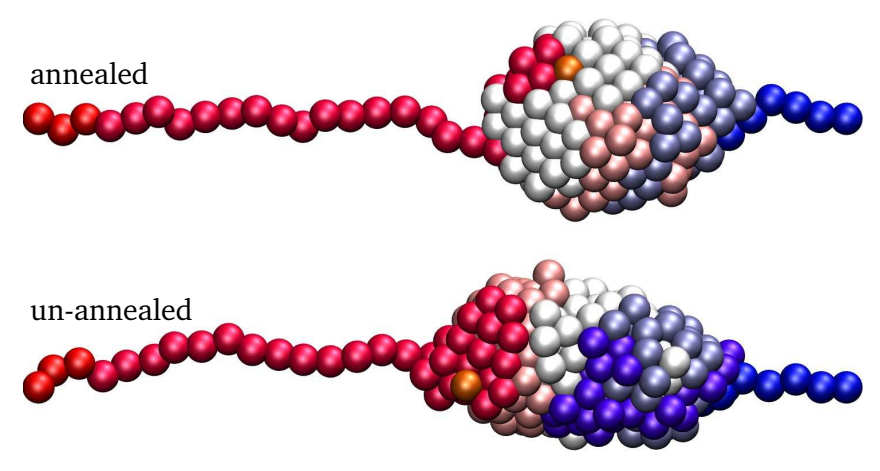

Figure 9: Typical initial configurations in the annealed (top) and un-annealed simulations (bottom) for $\tilde{\varepsilon}=2.91$ and $\tilde{v}=0.001$. For the large $\varepsilon$ shown here, the collapsed un-annealed structures show some residual ordering. The color coding indicates the monomer index along the chain contour.

\subsection{Liquid-solid transition for large cohesive strengths}

In fig. 10, stretching curves averaged over twenty pulling cycles for the annealed pulling protocol are shown for various cohesive strengths $\varepsilon$ and $\tilde{v}=0.001, N=300$. Beyond the globule transition, $\tilde{\varepsilon}>\tilde{\varepsilon}_{\mathrm{G}} \approx 0.5$ [32, 43, 60], an $\varepsilon$ dependent force plateau is observed. The plateau force $F_{\mathrm{p}}$ increases as $\varepsilon$ increases and is, for the relatively slow pulling speed shown here, mostly associated with the equilibrium free energy per unit length of globule formation, $F_{\mathrm{p}} a \sim \varepsilon-\varepsilon_{\mathrm{G}} \underline{32}$. For large extensions of the order of $x / L_{\mathrm{c}} \approx 0.8$, a dip in the force extension curve appears, which is the signature of a pulling induced globule dissolution. For even larger extension, essentially no monomer contacts are present, and the force extension curve becomes independent of the cohesive strength $\varepsilon$ and follows the trace of an extensible freely jointed chain (shown as a broken line)

$$
x / L_{\mathrm{c}}=\operatorname{coth}\left(2 a F /\left(\mathrm{k}_{\mathrm{B}} T\right)\right)+\mathrm{k}_{\mathrm{B}} T /(2 a F)+F /(2 a \kappa) .
$$

A phantom chain $(\tilde{\varepsilon}=0)$ coincides perfectly with eq. 20 . The curve for $\tilde{\varepsilon}=2.5$ features a maximum at $x / L_{\mathrm{c}} \approx 0.3$, whose origin will be discussed in the next paragraph.

Fig. 11 shows force extension traces for the two different pulling protocols for $N=300, \tilde{v}=0.001$, and relatively strong cohesive forces $\tilde{\varepsilon}=2.08,2.5,2.91$. The thick curve in each plot depicts the average over all 20 pulling curves, while the thin curves show individual force-extension traces for different initial configurations. In figs. 11a-c pulling curves starting from un-annealed configurations are shown. Increasing $\varepsilon$ leads to an increasing plateau force similar to fig. 10. For $\tilde{\varepsilon} \geq 2.5$ stronger fluctuations of the force are observed, but the force extension traces are qualitatively 


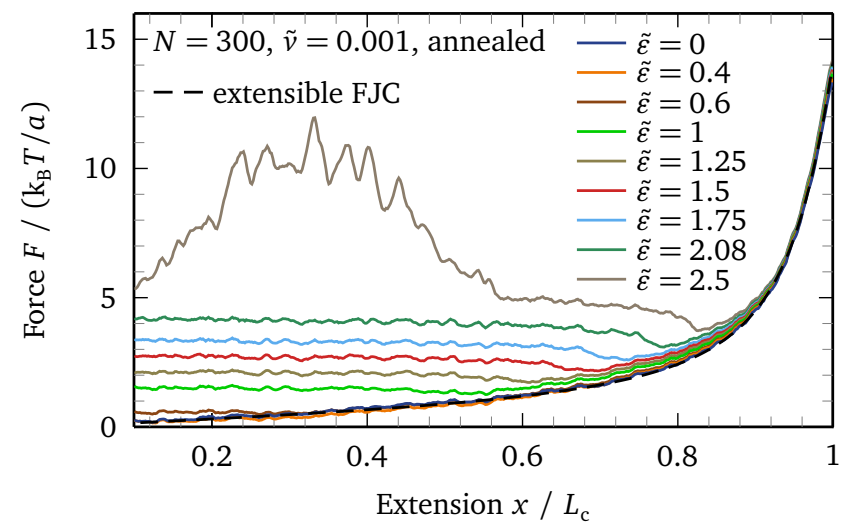

Figure 10: Force extension curves for constant velocity $\tilde{v}=0.001$, various cohesive strengths and $N=300$ using annealed initial configurations. All curves are averages over twenty pulling cycles. Above the globule transition, $\tilde{\varepsilon}>$ $\tilde{\varepsilon}_{\mathrm{G}} \approx 0.5$, a force plateau followed by a dip in the force extension curve is observed. The broken line depicts the theoretically expected force extension trace of an extensible freely jointed chain for $\tilde{\varepsilon}=0$, eq. (20). For $\tilde{\varepsilon}=2.5$ the pulling curve exhibits a marked maximum at small extensions, which indicates frozen internal dynamics.

similar to the curves for $\tilde{\varepsilon}=2.08$. The situation is vastly different for pulling curves starting from the annealed initial configurations, figs. 11 d-f. Again, for small cohesive strengths $\tilde{\varepsilon} \leq 2.08$ the pulling curves are smooth and no strong fluctuations occur, cf. fig. 10. However, increasing the cohesive strength further leads to pronounced fluctuations in the force extension curves. This is due to a transition of the internal dynamics from liquid-like to solid-like [32, 42, 45, 4850, 61. Since the un-annealed initial configurations are rather ordered - especially for large $\varepsilon-$ the globules are easily unwound by simply retracing the configurational intermediates that were encountered upon folding in reversed order. The liquid-solid transition occurs for the un-annealed structures, too, but has almost no effect on the non-equilibrium pulling simulations. This is very different for the annealed simulation protocol, where the mean force is higher and the variation of individual force curves around the mean force is also pronounced.

The dependence of the liquid-solid transition on the globule size $N_{\mathrm{G}}$ is illustrated in fig. 12 . Here, force curves obtained using the annealed pulling protocol are plotted versus the number of monomers $N_{\mathrm{G}}$ inside the globule for different monomer numbers $N$ and cohesive strengths. $N_{\mathrm{G}}$ is calculated via a modified version of eq. 12 where the extension $x$ of the polymer is used instead of $L$. One notices that the pronounced noise in the curves ceases once the globule is below a certain size, i.e. once $N_{\mathrm{G}}$ is below a certain threshold value, where this critical size depends on $\varepsilon$. This feature is independent of the chain length $N$ but solely depends on $N_{\mathrm{G}}$, i.e. curves with equal $\varepsilon$ but different $N$ coincide once the globule size has fallen under the critical size 42,50 . The cohesive strength $\varepsilon_{\mathrm{s}}$ at which this liquidsolid transition occurs is also independent of the pulling velocity within the range of velocities studied. In fig. 13 we see that for $\tilde{\varepsilon}=2.08$ even with the highest pulling velocity, no large fluctuations in the force extension curves are induced and the globule remains in the liquid phase. Therefore, the liquid-solid transition at $\varepsilon_{\mathrm{S}}$ is not a mere non-equilibrium pulling feature but an indication of a change in the equilibrium internal dynamics. The transition observed in the non-equilibrium pulling simulations is of course the same transition that induces the abrupt change of the equilibrium globule diffusivity, demonstrated in fig. 6, since both the equilibrium globule diffusion and the non-equilibrium pulling response are ultimately related to the configurational chain dynamics inside the globule.

\subsection{Internal friction}

\subsubsection{Internal friction is viscous}

For small $\varepsilon<\varepsilon_{\mathrm{s}}$, the internal chain dynamics is fast and no significant difference between the simulations with annealed and un-annealed initial configurations is observed. In fig. 13 pulling curves for various pulling velocities $v$ are shown. We observe that even for a cohesive strength as large as $\tilde{\varepsilon}=2.08$ and polymers as long as $N=300$, the two slowest pulling curves $\tilde{v}=0.001,0.0045$ almost coincide. This indicates that force extension curves for $\tilde{v}=0.001$ are already very good approximations for equilibrium curves. Increasing velocity leads to increasing energy dissipation. There are two major mechanisms leading to dissipation: solvent friction and internal friction. These dissipation mechanisms dominate in different parts of the pulling curve. For small extensions, when most of the monomers are part of the globule, internal friction dominates. Towards the end of the pulling process, the globule is markedly smaller and the 


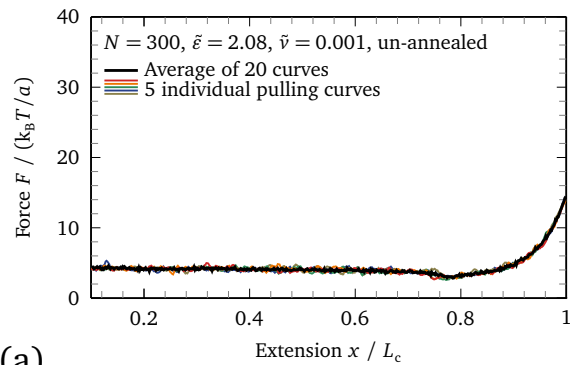

(a)

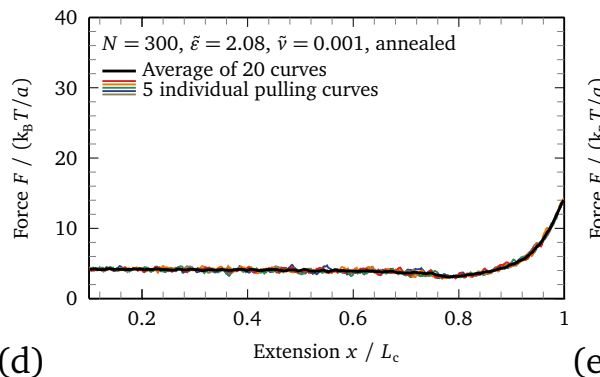

(e)

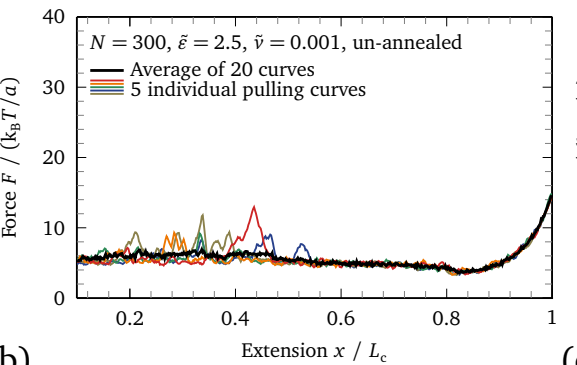

(b)

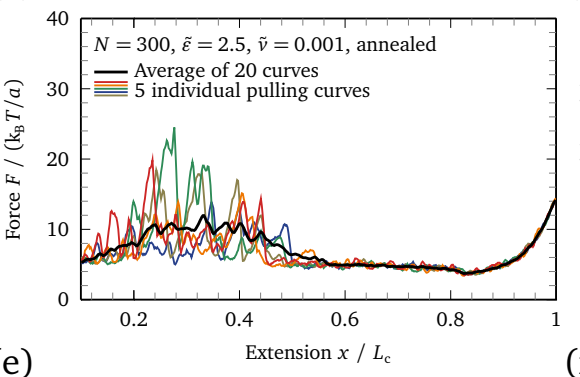

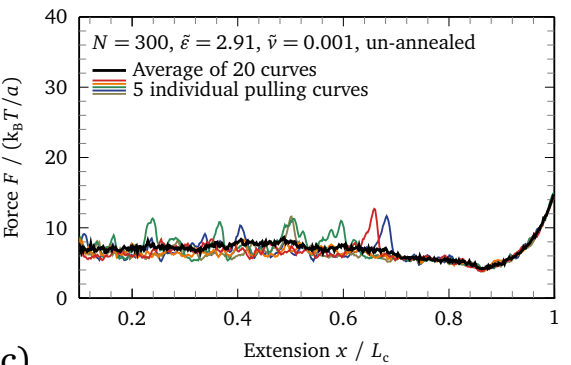

(c)

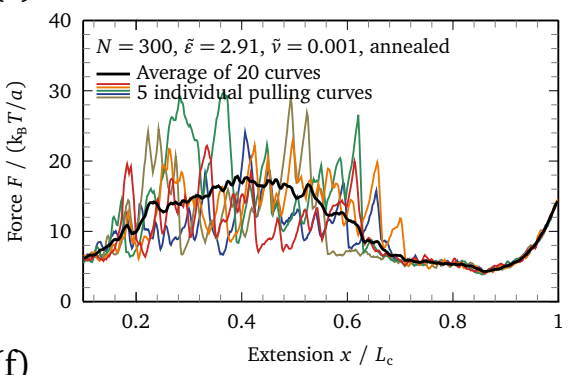

Figure 11: Pulling curves for $N=300$ and $\tilde{v}=0.001$ with (a-c) un-annealed and (d-f) annealed structures as initial configurations. The thin lines are individual force extension traces, whereas the thick black line is the average over twenty pulling curves. The un-annealed structures exhibit rather smooth pulling curves and no drastic differences between the various cohesive strengths $\varepsilon$ is observed. In contrast, the annealed structures feature marked fluctuations above a certain threshold $\tilde{\varepsilon}_{\mathrm{s}} \approx 2.1$ for $N=300$, which diminish for large extension, i. e. when the globule sizes decrease. We attribute these fluctuations to a liquid-solid transition, which dramatically changes the internal dynamics (see text).

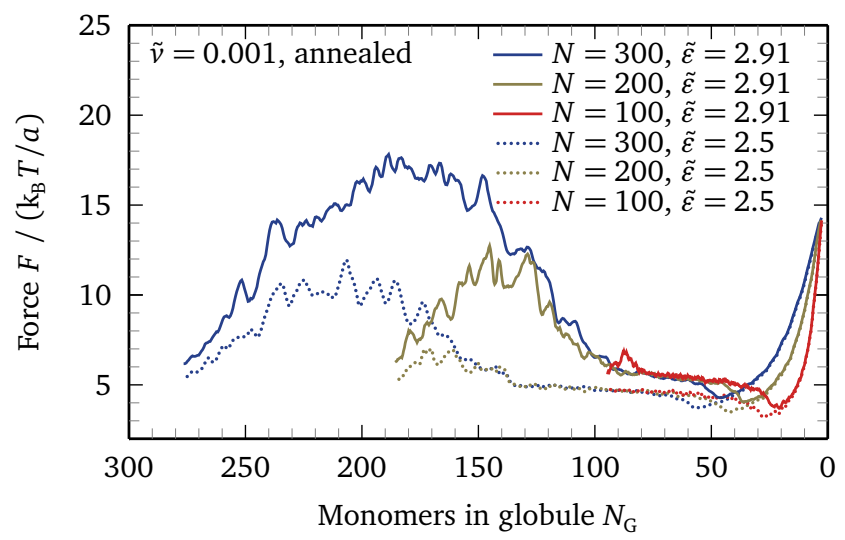

Figure 12: Averaged force extension curves in the vicinity of the liquid-solid transition are shown as a function of the number of monomers inside the globule, $N_{\mathrm{G}}$, eq. $(12)$, for the annealed set of initial configurations. Once $N_{\mathrm{G}}$ is below a certain threshold $(\approx 100$ for $\tilde{\varepsilon}=2.91, \approx 140$ for $\tilde{\varepsilon}=2.5)$ the globule is driven into the liquid state, and force curves for different $N$ and equal $\varepsilon$ collapse. The dependence of the liquid-solid transition on $N_{\mathrm{G}}$ is discussed in detail in ref. 42 .

solvent friction, which acts mostly on the linker chain, dominates the pulling curve. The dissipated work $\Delta W(v, \varepsilon)$ is defined as the difference between the total work at finite velocity and the equilibrium work 32 .

$$
\Delta W(v, \varepsilon)=W(v, \varepsilon)-W_{\mathrm{eq}}(\varepsilon),
$$

where the work done by one trap is generally defined as

$$
W=-\int_{R_{x}^{(\min )}}^{R_{x}^{(\max )}} F(x) \mathrm{d} x,
$$

see the inset of fig. 13 for an illustration of this definition. The equilibrium work $W_{\text {eq }}(\varepsilon)$ is obtained from extrapolating $W(v, \varepsilon)$ to $v \rightarrow 0$. In fig. 14 we show the dissipated work per monomer, $\Delta W / N$, as a function of the velocity $v$ for 


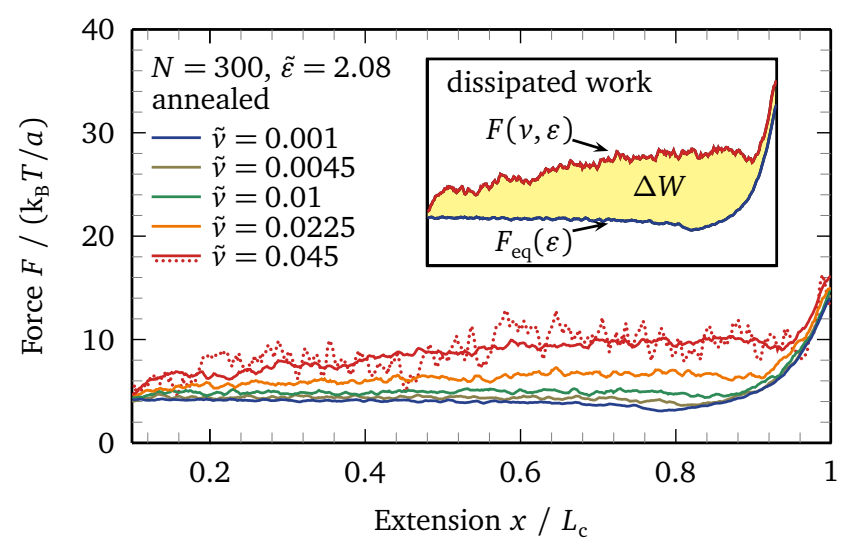

Figure 13: Averaged pulling curves for various pulling velocities with $N=300, \tilde{\varepsilon}=2.08$, and annealed initial configurations. The friction force increases with increasing pulling velocity. The curves with the slowest pulling velocities $\tilde{v}=0.001,0.0045$ almost coincide, indicating that with these slow velocities one has approximately reached the equilibrium pulling limit. The dotted curve is one individual pulling curve for $\tilde{v}=0.045$. The inset illustrates the definition of the dissipated work, eq. 21), which is the shaded area between the two curves.

$$
\begin{array}{l|cccc}
N & 100 & 150 & 200 & 300 \\
\hline \tilde{\varepsilon}_{\mathrm{s}} & 4 & 2.9 & 2.3 & 2.1
\end{array}
$$

Table 1: The critical cohesion strength $\varepsilon_{\mathrm{s}}$ of the liquid-solid transition depends on the length of the polymer $N$. For $\varepsilon<\varepsilon_{\mathrm{s}}$, the system is in the liquid phase and fluctuations of the dissipated work are small. For $\varepsilon>\varepsilon_{\mathrm{s}}$ pronounced fluctuations are observed due to slow internal dynamics, see fig. 16.

$N=300$ and various $\varepsilon$. Below the liquid-solid transition, $\tilde{\varepsilon}<\tilde{\varepsilon}_{\mathrm{s}} \approx 2.1$ for $N=300$, the dissipated work scales linearly with $v$, which is further demonstrated in fig. 15, where the ratio $\Delta W /(v N)$ is plotted. This shows that in the liquid state, the simulations are conducted in the experimentally relevant linear response regime and the friction is essentially of viscous nature. Above the liquid-solid transition this scaling breaks down, or, in other words, the velocities probed in the simulations are not low enough to reach the viscous regime. Further, we observe that below the globule transition, $\tilde{\varepsilon}<\tilde{\varepsilon}_{\mathrm{G}} \approx 0.5$, the dissipated work is almost independent of the cohesive strength. This suggests that monomer-monomer attraction and topological constraints, e.g. entanglements, are negligible for small values of the cohesive strength, i.e. in the non-collapsed state. In order to extract such subtle friction effects, much longer simulations would be needed. Knots, which in principle might arise due to bond crossing, are not observed in our simulations.

The fluctuations of the dissipated work can be conveniently used to define the liquid-solid transition. In fig. 16 the standard deviation

$$
\sigma_{\Delta W}=\sqrt{k /(k-1)} \sqrt{\left\langle\Delta W^{2}-\langle\Delta W\rangle^{2}\right\rangle}
$$

obtained from $k=20$ measurements of $\Delta W$ for $\tilde{v}=0.001$ in the annealed pulling protocol is plotted against $\varepsilon$ on both linear and logarithmic scales. For $\varepsilon<\varepsilon_{\mathrm{s}}, \sigma_{\Delta W} / N$ is independent of $N$ and $\varepsilon$. As one crosses from the liquid into the solid phase at $\varepsilon=\varepsilon_{\mathrm{s}}$, the fluctuations increase. Fig. 16 clearly demonstrates that $\varepsilon_{\mathrm{s}}$ depends on the system size $N$. The estimates of $\varepsilon_{\mathrm{s}}$ extracted from the behavior of $\sigma_{\Delta W} / N$ are shown in tab. 1 .

\subsubsection{Internal friction is extensive}

We demonstrated that in the liquid state the dissipated work $\Delta W$ and thus the internal friction scale linearly with the velocity $v$. Therefore, we can express the friction force $F_{\text {fr }}$ in terms of a velocity independent viscous friction coefficient $\Gamma\left(\varepsilon, N_{\mathrm{G}}\right)$, which depends on the number of monomers in the globule,

$$
F_{\mathrm{fr}}\left(v, \varepsilon, N_{\mathrm{G}}\right)=F\left(v, \varepsilon, N_{\mathrm{G}}\right)-F_{\mathrm{eq}}\left(\varepsilon, N_{\mathrm{G}}\right)=v \Gamma\left(\varepsilon, N_{\mathrm{G}}\right),
$$

where $F_{\text {eq }}$ is the equilibrium force extension curve. Analogous to the Stokes friction of a sphere, we define the friction coefficient as 32

$$
\Gamma\left(\varepsilon, N_{\mathrm{G}}\right) \sim a \eta_{\mathrm{G}}(\varepsilon) N_{\mathrm{G}}^{\gamma}
$$




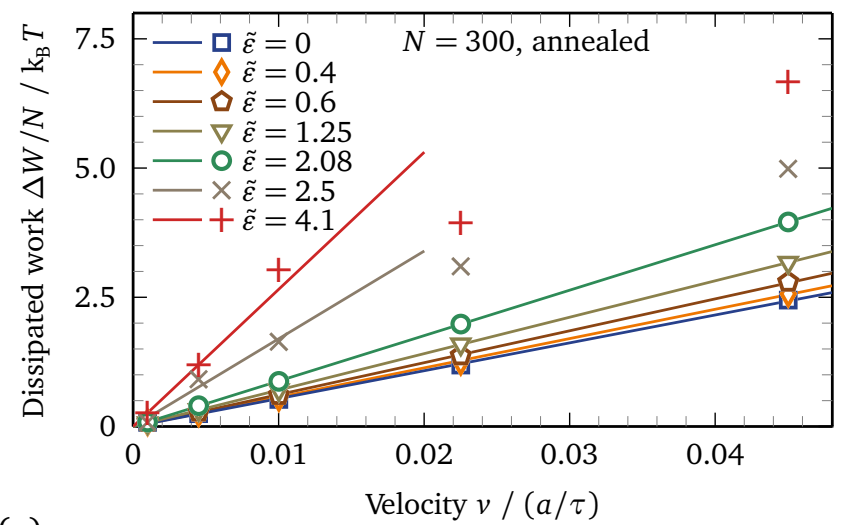

(a)

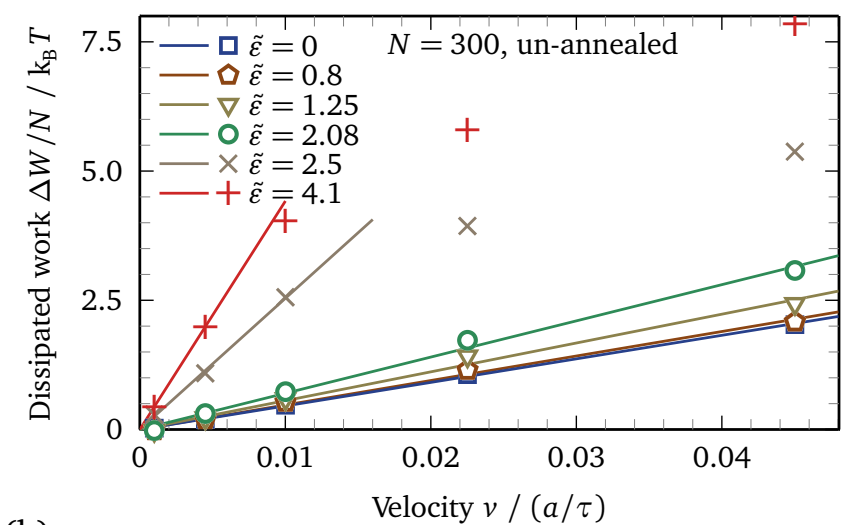

(b)

Figure 14: Dissipated work per monomer $\Delta W / N$ as a function of the pulling velocity $v$ for $N=300$ and different cohesive strengths $\varepsilon$ starting from the (a) annealed and the (b) un-annealed set of initial configurations. Symbols depict simulation data, lines show linear fits to the data according to eq. (27). Below the globule transition, $\tilde{\varepsilon}<\tilde{\varepsilon}_{\mathrm{G}} \approx 0.5$, the curves collapse, i. e. interaction contributions to friction are negligible. Below the liquid-solid transition, $\tilde{\varepsilon}<\tilde{\varepsilon}_{\mathrm{s}} \approx 2.1$ for $N=300$, the data is linear in $v$ in the whole range of velocities studied, indicating that friction is of viscous nature. Above the liquid-solid transition the linear scaling breaks down. $\Delta W$ is slightly lower for the simulations starting from the un-annealed set of initial configurations showing that the globule is not completely equilibrated and still rather ordered.

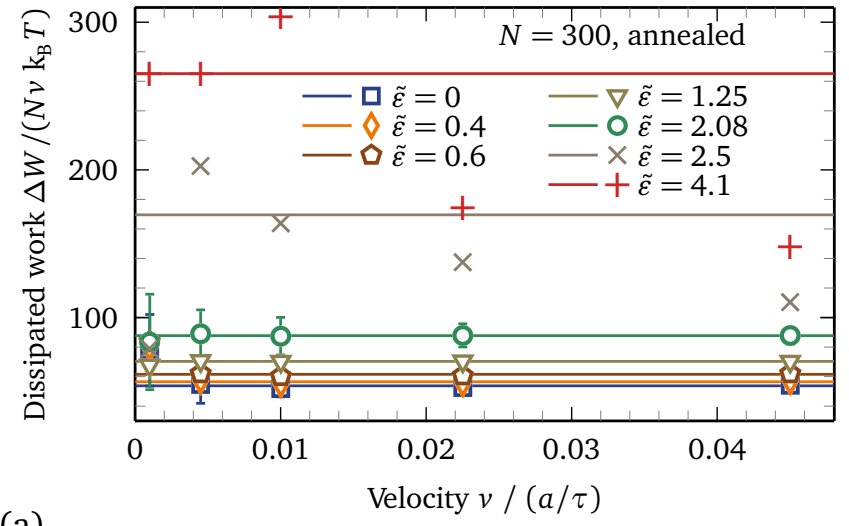

(a)

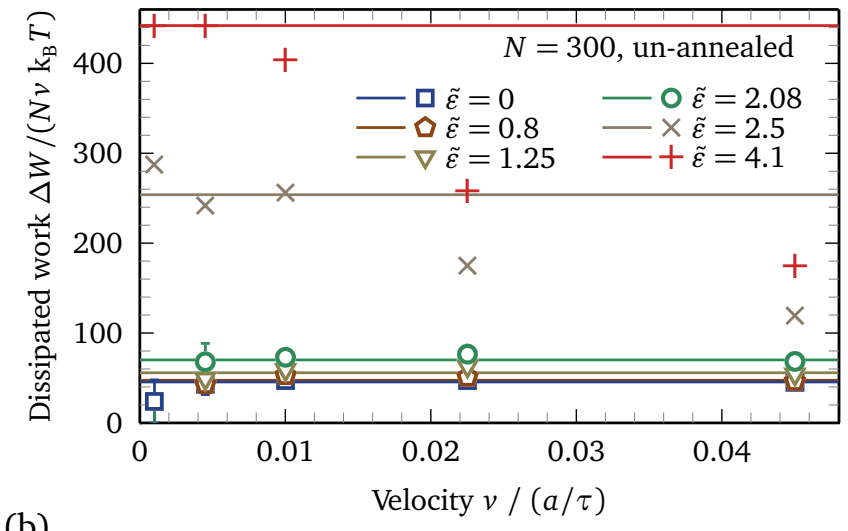

(b)

Figure 15: Dissipated work per monomer rescaled by the pulling velocity, $\Delta W /(N v)$, as a function of the pulling velocity $v$ for $N=300$ and different cohesive strengths $\varepsilon$ starting from the (a) annealed and the (b) un-annealed set of initial configurations. Symbols denote simulation results, lines depict $\tilde{\Gamma}$ as obtained from linear fits according to eq. 27] in fig. 14. For $\varepsilon<\varepsilon_{\mathrm{s}}$, our simulations are carried out in the linear viscous regime and there is no $v$ dependence after rescaling. Above the liquid-solid transition the linear scaling in $v$ breaks down. For $\tilde{\varepsilon}=0$ and 2.08 , error bars indicate the standard deviations of the twenty measurements of $\Delta W(v, \varepsilon)$.

where $\eta_{\mathrm{G}}(\varepsilon)$ is the internal viscosity, which depends on $\varepsilon$ but not on $N_{\mathrm{G}}$. The exponent $\gamma$ describes the dependence on the monomer number inside the globule, and characterizes the friction mechanism at work during unraveling the globule. Two limits can be distinguished: first, if $\gamma=0$, the friction force is independent of the globule size $N_{\mathrm{G}}$, and only a finite number of monomers, which does not scale with $N_{\mathrm{G}}$, contribute to dissipation. We call this limit local or intensive friction, and the pictorial mechanism for such a scenario could be that monomers are peeled off one-by-one from the surface of the globule. Second, $\gamma=1$ describes the limiting situation where a finite fraction of the globule that is proportional to $N_{\mathrm{G}}$, or even the entire chain, rearranges in the unfolding process and hence contributes to the friction force. We call this limit global or extensive friction and note that there are several conceivable microscopic mechanisms. One possibility could be a reptation scenario, where a reorganization of the globule conformation is accompanied by a reptation of the entire globular chain section (or a chain fraction of length proportional to $N_{\mathrm{G}}$ ) through the globule. Another possibility would be that the stress propagates through the whole globule and leads to 


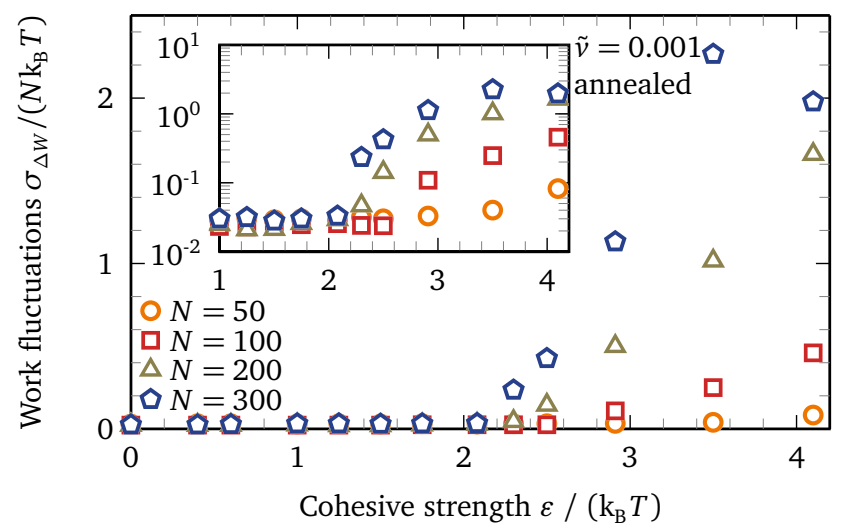

Figure 16: The standard deviation per monomer $\sigma_{\Delta W} / N$ of the dissipated work, as obtained from twenty pulling cycles. For $\varepsilon<\varepsilon_{\mathrm{s}}, \sigma_{\Delta W} / N$ is independent of the chain length $N$ and small. The fluctuations of the dissipated work increase significantly when $\varepsilon>\varepsilon_{\mathrm{s}}$, where $\varepsilon_{\mathrm{s}}$ decreases for increasing $N$, see tab. 1 . The data is obtained from simulations with $\tilde{v}=0.001$ and annealed initial configurations.

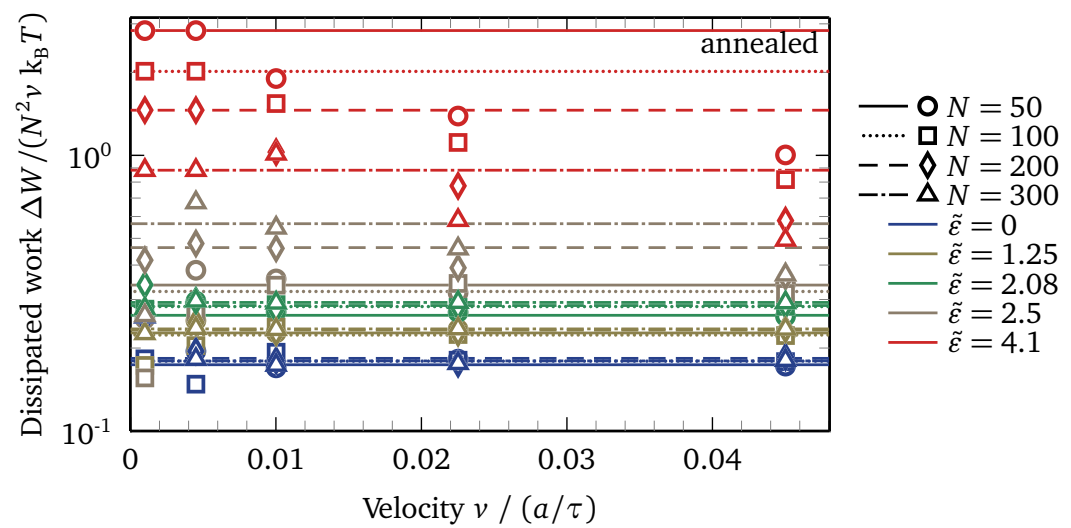

Figure 17: Rescaled dissipated work per monomer $\Delta W /\left(N^{2} v\right)$ as a function of the pulling velocity $v$ for different $N$ and cohesive strengths $\varepsilon$ starting from the annealed set of initial configurations. Curves with equal $\varepsilon$ and different $N$ collapse for $\varepsilon<\varepsilon_{\mathrm{s}}$. This shows that the dissipated work per monomer is an extensive function and $\Delta W \sim N^{2} v$. Again, this scaling behavior breaks down above the liquid-solid transition.

dissipation without an actual reptation of the chain. We did not analyze the precise microscopic dissipation mechanism and therefore leave this issue for future work.

Integrating the friction force, eq. 24, yields the scaling form of the dissipated work

$$
\Delta W(v, \varepsilon, N)=-\int F_{\mathrm{fr}}\left(v, \varepsilon, N_{\mathrm{G}}\right) \mathrm{d} x \sim \eta_{\mathrm{G}}(\varepsilon) a^{2} N^{\gamma+1} v
$$

where we assume $N_{\mathrm{G}} \approx\left(L_{\mathrm{c}}-x\right) /(2 a)$. In fig. $17 \Delta W / N^{2}$ is plotted versus $v$, the data with equal $\varepsilon<\varepsilon_{\mathrm{s}}$ and different $N$ collapse. This implies that the dissipated energy per monomer $\Delta W / N$ and also the internal friction is extensive, $\gamma=1$, meaning that a finite fraction of the globule or the whole globule rearranges during pulling and not only a few monomers. This scaling behavior breaks down above the liquid-solid transition, which however does not imply that the friction becomes non-extensive but rather that linear viscous scaling is not valid anymore. It is also not clear whether the extensive scaling of the viscosity, defined here via the viscous force when pulling a chain segment out of the globule, should be expected to persist in the limit of very large globules, as the scaling suggests the viscosity in fact to diverge in this thermodynamic limit. More work on this is also needed. 


\subsubsection{Internal viscosity}

To extract the internal viscosity quantitatively, we fit the dissipated work to a linear form in $v$ according to

$$
\Delta W(v, \varepsilon, N)=\tilde{\Gamma}(\varepsilon, N) v
$$

and extract the prefactor $\tilde{\Gamma}(\varepsilon, N)$, which is apart from geometrical prefactors proportional to the friction coefficient $\Gamma$ defined in eq. (24). As can be seen in fig. 14. $\Delta W \sim v$ for all velocities studied if $\varepsilon<\varepsilon_{\mathrm{s}}$. Consequently we include all velocity data in the linear fit. However, for $\varepsilon>\varepsilon_{\mathrm{s}}$ a marked deviation from linear behavior is observed. There, we fit only to the slowest velocities, where the data still scales linearly with $v$. From the scaling $\tilde{\Gamma}(\varepsilon, N) \sim \eta_{\mathrm{G}}(\varepsilon) N^{\gamma+1}$ one sees that the relative internal viscosity follows as

$$
\frac{\eta_{\mathrm{G}}(\varepsilon)}{\eta_{\mathrm{G}}(0)}=\frac{\tilde{\Gamma}(\varepsilon, N)}{\tilde{\Gamma}(0, N)},
$$

which is shown in fig. 18 for annealed and un-annealed initial configurations. Note that in the limit of vanishing cohesion, $\varepsilon=0$, the globular viscosity $\eta_{\mathrm{G}}(\varepsilon)$ is due to solvent friction effects only. The internal globular viscosity without solvent effects can therefore be defined by the difference $\eta_{G}(\varepsilon)-\eta_{G}(0)$, but note that this definition is approximate since the solvent friction also depends weakly on the value of $\varepsilon$ since the globular structure changes with varying cohesive strength. By plotting the ratio of the viscosity in the presence and absence of cohesion, any residual numerical prefactors and the polymer length dependence in eq. 26) are eliminated and we are able to compare all different sets of parameters. The internal viscosities extracted from the non-equilibrium pulling simulations coincide for different $N$, which indicates that the scaling Ansatz $\tilde{\Gamma}(\varepsilon, N) \sim \eta_{\mathrm{G}}(\varepsilon) N^{\gamma+1}$ works fine. In fig. 18 a for the annealed pulling simulations we add data for the internal viscosity obtained from the equilibrium globule diffusion simulations, which are defined via $\eta_{\mathrm{G}}(\varepsilon) / \eta_{\mathrm{G}}(0)=D_{0} / D_{\mathrm{G}}(\varepsilon)$ (the same data as already presented in fig. 60 b). The viscosity data from the equilibrium simulations are considerably lower for $\varepsilon<\varepsilon_{\mathrm{s}}$. This might in part be caused by an underestimate of the Rouse friction $D_{0}$ defined in eq. (16), which enters the definition of the viscosity in the equilibrium simulations, or by additional dissipative mechanisms in the non-equilibrium pulling simulations: sometimes it happens that the whole globule is moved through the solvent when an entanglement within the globule does not yield quickly enough, which causes additional solvent dissipation. Considering that the two ways of extracting the internal viscosity are very different, in terms of the geometry employed and the general setup (one being equilibrium, the other non-equilibrium), we consider the agreement sufficient at the present stage.

In fig. 18 we compare our data to a simple model based on the effective friction coefficient of a single particle diffusing in the periodic potential $U_{\mathrm{p}}=(\theta \varepsilon / 2) \cos (\pi x / a)$ with amplitude $\theta \varepsilon$. This potential mimics the energy landscape the chain monomers are experiencing as they are moving against each other during conformational reorganization processes. In writing the amplitude of the potential as $\theta \varepsilon$, we assume the corrugation strength to be proportional to the cohesive energy $\varepsilon$. The numerical prefactor $\theta$ is a scaling factor that may be viewed as a fitting parameter. The solution of this one-dimensional diffusion problem yields an effective viscosity [30]

$$
\frac{\eta(\varepsilon)}{\eta(0)}=\mathrm{I}_{0}^{2}\left(\frac{\theta \varepsilon}{2 \mathrm{k}_{\mathrm{B}} T}\right)
$$

in the stationary long-time limit. $\mathrm{I}_{0}(z)$ is the zeroth order modified Bessel function with the asymptotic limits $\mathrm{I}_{0}(z) \sim$ $1+z^{2} / 4$ for $z \ll 1$ and $\mathrm{I}_{0}(z) \sim \mathrm{e}^{z} / \sqrt{2 \pi z}$ for $z \gg 1$ 62. As can be seen from figs. 18, the rescaled internal viscosity from simulations, as defined in eq. (28), is reproduced quite well by the model prediction eq. $(29)$ with a fitting value $\theta=1$, which is shown by a solid line. Our results compare excellently to our previous results where smaller globules $N \leq 100$ have been considered [32].

In the solid regime for large cohesive energies $\varepsilon>\varepsilon_{\mathrm{s}}$, pronounced deviations between the equilibrium diffusional and the non-equilibrium pulling simulations appear, which are better appreciated when the whole data set is plotted on a logarithmic scale in fig. 18k. The pulling simulations give a much smaller viscosity when compared to the equilibrium diffusion simulations, which shows that the pulling simulations for $\varepsilon>\varepsilon_{\mathrm{S}}$ do not reach the linear response regime, a fact that is independently suggested by the absence of scaling in fig. 14

\subsection{Summary for pulling on homopolymer globules}

The dissipated work per monomer scales linearly in the pulling velocity, indicative of viscous friction; the friction is also proportional to the number of monomers in the globule and scales extensive, thus a finite fraction of the globule contributes to the internal friction, fig. 17. We show that the dependence of the internal friction on the cohesive strength $\varepsilon$ is described well by the diffusion of a single particle in a corrugated potential. We extend our previous results 32 to significantly larger systems and are able to show that - below the liquid-solid transition - the history, i. e. the preparation of the initial structures, does not influence the scaling results, see fig. 18 


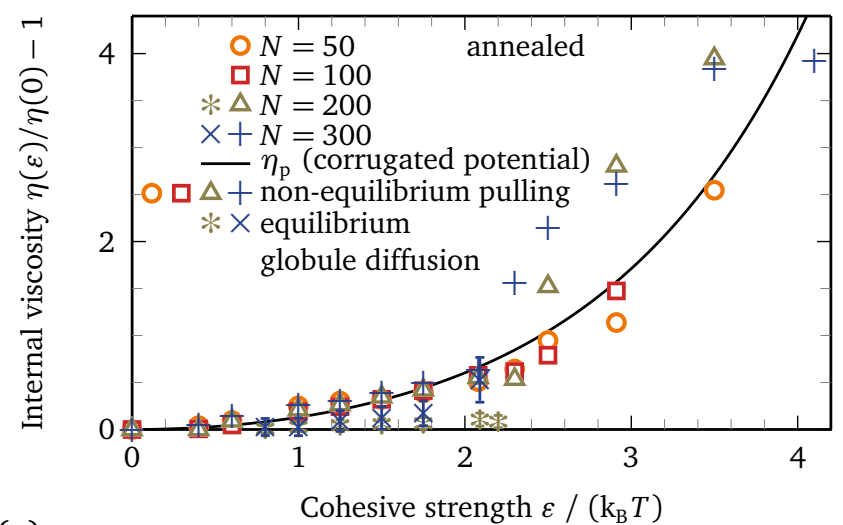

(a)

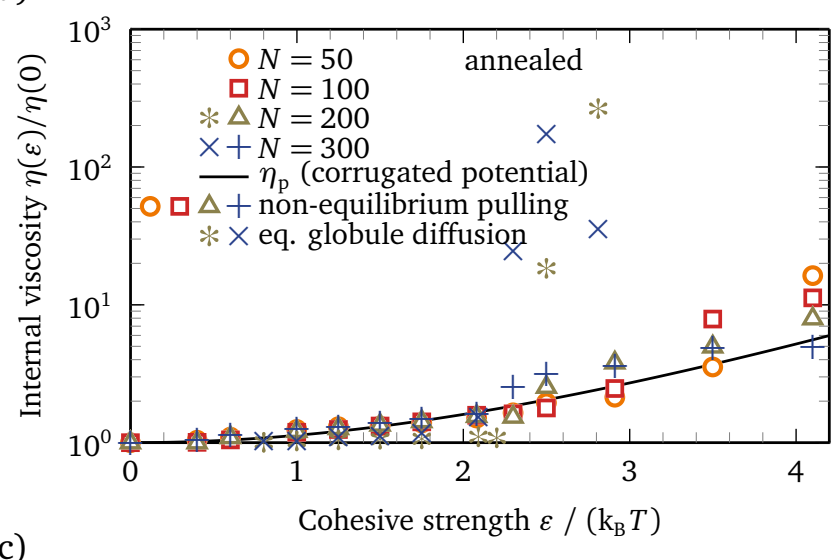

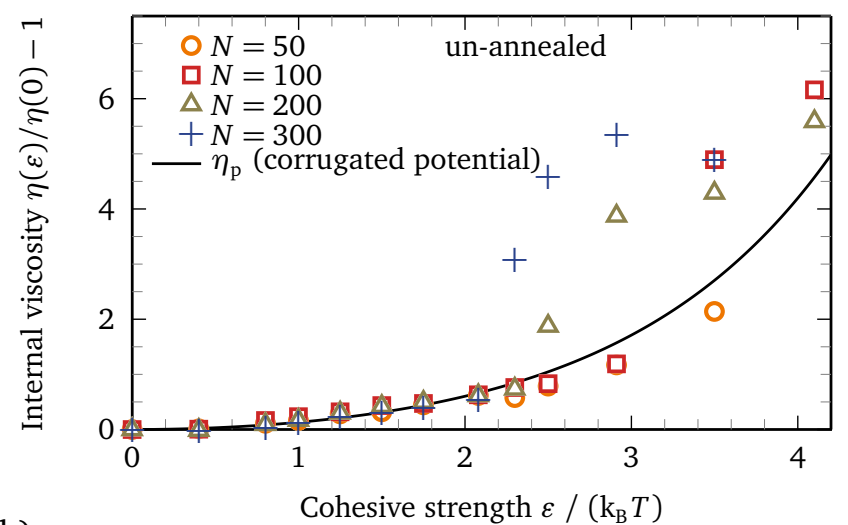

(b)

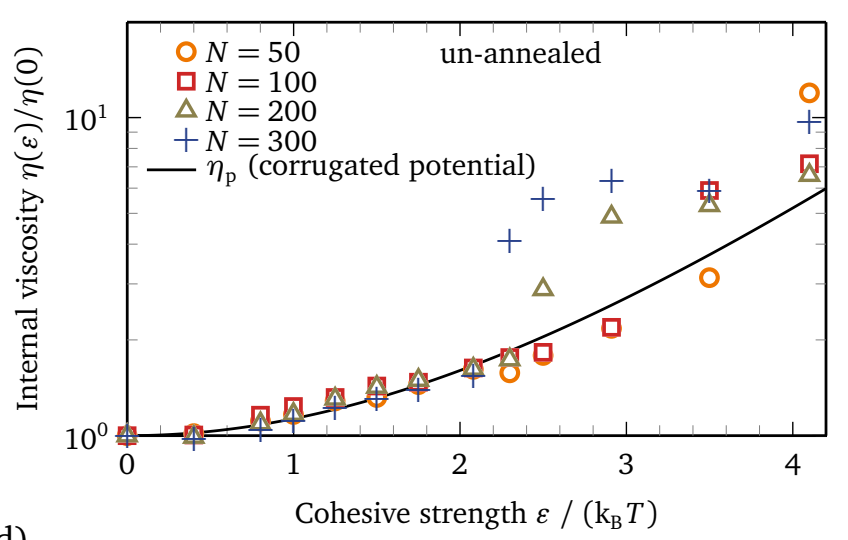

Figure 18: Rescaled internal viscosity $\eta_{\mathrm{G}}(\varepsilon) / \eta_{\mathrm{G}}(0)$, eq. (28), as a function of the cohesive strength $\varepsilon$ for (a), (c) annealed (b), (d) un-annealed initial configurations. The viscosity $\eta_{G}(\varepsilon)$ is obtained from linear fits to the dissipated work as a function of the velocity $v$. As the data points for different $N$ coincide, all $\tilde{\Gamma}(\varepsilon, N)$ exhibit the same $N$ dependence. The solid line is the prediction of the excess viscosity of a Brownian particle in a sinusoidal potential, see eq. (29). In (a), (c) the viscosity ratio as obtained from our equilibrium simulations, see fig. $6 \mathrm{p}, \eta_{\mathrm{G}}(\varepsilon) / \eta_{\mathrm{G}}(0)=D_{0} / D_{\mathrm{G}}(\varepsilon)$ is shown in addition.

\section{Summary and conclusions}

Two different dynamical regimes exist for a globular homopolymer, a solid-like regime at large cohesive energies or low temperatures, characterized by a substantially increased force needed to unfold the globule and by pronounced fluctuations in the force extension traces, and a liquid-like regime at low cohesive energies or high temperatures. The critical cohesive energy $\varepsilon_{\mathrm{s}}$ at the transition between these two regimes depends on the size of the globule $N_{\mathrm{G}}$, as shown in figs. 12 and 16. In the liquid regime the monomers inside the globule are rather mobile and the internal friction or viscosity in the globule can be extracted from simulations. The globule dynamics is studied by two different scenarios, (i) by considering the equilibrium diffusion of a globule relative to the linking straight chain sections, and (ii) by non-equilibrium stretching simulations. In both scenarios we find that the internal friction or viscosity is extensive, thus scaling linearly with $N_{\mathrm{G}}$ and increases with growing $\varepsilon$ until the liquid-solid transition is reached. The signature of the solid state is a vanishing globule diffusion on the simulation time scales, $D_{\mathrm{G}}=0$, for the equilibrium globule diffusion setup, while huge fluctuations in the force extension curves are seen for the non-equilibrium pulling setup. The solid state is characterized by very slow internal dynamics and no reliable estimates for the internal friction can be obtained from our simulations.

Note that we define the internal viscosity in a rather specialized way, namely via the viscous force needed to pull a chain segment out of the globule (other definitions of internal viscosity are possible and useful in different contexts). Our prediction that the friction of chain motion scales extensively with the globular size should be discussed in light of the classical reptation scenario [8]: Here, the friction coefficient (per monomer) is assumed to be independent of the globule size but rather the whole chain is assumed to move or reptate through the melt, also giving rise to an extensive scaling of the friction. This suggests that reptation is at the heart of the extensive scaling of the viscosity found in our simulations, but other mechanisms are also conceivable. Besides, it is not clear whether the extensive scaling of the 
viscosity holds also in the thermodynamic limit and how a possible crossover is brought about. For a melt consisting of finite-length polymers, the chain size would constitute a possible crossover length, for the hypothetical limit of a single infinitely long polymer chain no such crossover length comes easily to mind.

The most direct experimental realization of our system would be possible with a homopolymeric globule in a singlemolecule setup. But there might also be implications for protein folding. Our results suggest that a quick collapse of a protein might result in a kinetically trapped and misfolded hydrophobic core, a so-called molten globular state, which would take a long time to fold into the native structure due to high internal friction. Since the friction scales extensively with the monomer number inside the globular core, the folding time becomes prohibitively long already for moderate globule size. From solvent-viscosity dependent measurements of folding times, the internal viscosity of a short $\alpha$-helix forming peptide in the absence of solvent viscosity effects has been estimated to be of the same order as the solvent viscosity, i.e. $\eta_{\mathrm{G}}(\varepsilon) / \eta_{\mathrm{G}}(0) \simeq 2$ in our notation 9 . The interaction energy we extract from fig. 18 follows to be of the order of $\varepsilon \simeq 2.5 \mathrm{k}_{\mathrm{B}} T$, not unrealistic for typical interaction parameters for residue-residue contacts. When speculating about the relevance of our results to protein folding, a few cautious remarks are in order: First, protein collapse is driven by the hydrophobic effect, which is quite involved due to the presence of water solvent, neglected in our treatment using an implicit solvent model with Lennard-Jones interactions. We would think that the scaling properties of the internal friction and internal viscosity will not change by adding explicit solvent, which however should be tested. Secondly, specific interactions between protein residues, sequence effects and the existence of a well defined native state will certainly alter the friction behavior as well. One lesson that might be learned from our work is that even for the relatively simple case of a homopolymeric globule, the concepts of internal friction and internal viscosity are far from trivial.

\section{Acknowledgements}

The authors would like to thank the DFG for financial support via grants NE 810/8 and SFB 863 and the Leibniz Rechenzentrum for providing computing facilities. T.R.E. acknowledges support from the Elitenetzwerk Bayern within the framework of CompInt, C.E.S. acknowledges support from the NDSEG fellowship.

\section{References}

1. J. Chuang, Y. Kantor, M. Kardar, Phys. Rev. E 65(1), 011802 (2001)

2. R. Bundschuh, U. Gerland, Phys. Rev. Lett. 95, 208104 (2005)

3. K. Luo, T. Ala-Nissila, S.C. Ying, R. Metzler, Europhys. Lett. 88(6), 68006 (2009)

4. N. Gunari, A.C. Balazs, G.C. Walker, J. Am. Chem. Soc. 129(33), 10046 (2007)

5. M. Rief, M. Gautel, F. Oesterhelt, J.M. Fernandez, H.E. Gaub, Science 276(5315), 1109 (1997)

6. D.B. Staple, S.H. Payne, A.L.C. Reddin, H.J. Kreuzer, Phys. Rev. Lett. 101(24), 248301 (2008)

7. M. Mandal, R.R. Breaker, Nat. Rev. Mol. Cell Biol. 5(6), 451 (2004)

8. P.G. de Gennes, J. Chem. Phys. 55(2), 572 (1971)

9. G.S. Jas, W.A. Eaton, J. Hofrichter, J. Phys. Chem. B 105(1), 261 (2001)

10. S.A. Pabit, H. Roder, S.J. Hagen, Biochemistry 43(39), 12532 (2004)

11. F. Graeter, P. Heider, R. Zangi, B.J. Berne, J. Am. Chem. Soc. 130(35), 11578 (2008)

12. D.E. Sagnella, J.E. Straub, D. Thirumalai, J. Chem. Phys. 113(17), 7702 (2000)

13. A.Y. Grosberg, S.K. Nechaev, E.I. Shakhnovich, J. Physique 49, 2095 (1988)

14. A. Grosberg, Y. Rabin, S. Havlin, A. Neer, Europhys. Lett. 23(5), 373 (1993)

15. E. Lieberman-Aiden, N.L. van Berkum, L. Williams, M. Imakaev, T. Ragoczy, A. Telling, I. Amit, B.R. Lajoie, P.J. Sabo, M.O. Dorschner et al., Science 326(5950), 289 (2009)

16. M.G. Poirier, A. Nemani, P. Gupta, S. Eroglu, J.F. Marko, Phys. Rev. Lett. 86(2), 360 (2001)

17. T. Frisch, A. Verga, Phys. Rev. E 65(4), 041801 (2002)

18. N.A. Denesyuk, J.D. Weeks, Phys. Rev. Lett. 102(10), 108101 (2009)

19. C.F. Abrams, N.K. Lee, S.P. Obukhov, Europhys. Lett. 59, 391 (2002)

20. A. Serr, C. Sendner, F. Müller, T.R. Einert, R.R. Netz, Europhys. Lett. 92, 38002 (2010)

21. F. Celestini, T. Frisch, X. Oyharcabal, Phys. Rev. E 70(1), 012801 (2004)

22. J. Chuang, A.Y. Grosberg, T. Tanaka, J. Chem. Phys. 112(14), 6434 (2000)

23. V. Barsegov, G. Morrison, D. Thirumalai, Phys. Rev. Lett. 100(24), 248102 (2008)

24. B.S. Khatri, M. Kawakami, K. Byrne, D.A. Smith, T.C.B. McLeish, Biophys. J. 92(6), 1825 (2007)

25. Y. von Hansen, F. Sedlmeier, M. Hinczewski, R.R. Netz (2010), to be published

26. A.E. Filippov, J. Klafter, M. Urbakh, Phys. Rev. Lett. 92(13), 135503 (2004)

27. Y. Murayama, H. Wada, M. Sano, Europhys. Lett. 79, 58001 (2007) 
28. A.V. Finkelstein, O.V. Galzitskaya, Phys. Life Rev. 1(1), 23 (2004)

29. R. Gerber, A. Tahiri-Alaoui, P.J. Hore, W. James, J. Biol. Chem. 282(9), 6300 (2007)

30. R. Zwanzig, Proc. Natl. Acad. Sci. U. S. A. 85(7), 2029 (1988)

31. C. Hyeon, D. Thirumalai, Proc. Natl. Acad. Sci. U. S. A. 100(18), 10249 (2003)

32. A. Alexander-Katz, H. Wada, R.R. Netz, Phys. Rev. Lett. 103(2), 028102 (2009)

33. M. Hinczewski, Y. von Hansen, J. Dzubiella, R.R. Netz, J. Chem. Phys. 132(24), 245103 (2010)

34. R.B. Best, G. Hummer, Proc. Natl. Acad. Sci. U. S. A. 107(3), 1088 (2010)

35. D.K. Klimov, D. Thirumalai, Proc. Natl. Acad. Sci. U. S. A. 96(11), 6166 (1999)

36. N. Yoshinaga, K. Yoshikawa, T. Ohta, Eur. Phys. J. E 17(4), 485 (2005)

37. G. Morrison, C. Hyeon, N.M. Toan, B.Y. Ha, D. Thirumalai, Macromolecules 40(20), 7343 (2007)

38. O. Braun, U. Seifert, Europhys. Lett. 68(5), 746 (2004)

39. R. Metzler, W. Reisner, R. Riehn, R. Austin, J.O. Tegenfeldt, I.M. Sokolov, Europhys. Lett. 76(4), 696 (2006)

40. A. Vologodskii, Biophys. J. 90(5), 1594 (2006)

41. L. Huang, D.E. Makarov, J. Phys. Chem. A 111(41), 10338 (2007)

42. C.E. Sing, T.R. Einert, R.R. Netz, A. Alexander-Katz (2010), manuscript in preparation

43. D.F. Parsons, D.R.M. Williams, Phys. Rev. E 74(4), 041804 (2006)

44. D.F. Parsons, D.R.M. Williams, J. Chem. Phys. 124, 221103 (2006)

45. W. Paul, T. Strauch, F. Rampf, K. Binder, Phys. Rev. E 75(6), 060801 (2007)

46. F. Rampf, W. Paul, K. Binder, Europhys. Lett. 70, 628 (2005)

47. Y. Zhou, C.K. Hall, M. Karplus, Phys. Rev. Lett. 77(13), 2822 (1996)

48. M.P. Taylor, W. Paul, K. Binder, J. Chem. Phys. 131, 114907 (2009)

49. V.G. Rostiashvili, G. Migliorini, T.A. Vilgis, Phys. Rev. E 64(5), 051112 (2001)

50. H. Liang, H. Chen, J. Chem. Phys. 113, 4469 (2000)

51. J. Torres, P. Nealey, J. de Pablo, Phys. Rev. Lett. 85, 3221 (2000)

52. T.S. Jain, J.J. de Pablo, Macromolecules 35(6), 2167 (2002)

53. F. Varnik, J. Baschnagel, K. Binder, Phys. Rev. E 65, 021507 (2002)

54. J. Baschnagel, F. Varnik, J. Phys.: Cond. Mat. 17(32), R851 (2005)

55. M. Doi, S.F. Edwards, The Theory of Polymer Dynamics (Clarendon Press, 1999)

56. W.T. Coffey, The Langevin Equation (World Scientific, 2005)

57. D.L. Ermak, J.A. McCammon, J. Chem. Phys. 69, 1352 (1978)

58. J.C.M. Gebhardt, T. Bornschlgl, M. Rief, Proc. Natl. Acad. Sci. U. S. A. 107(5), 2013 (2010)

59. M.P. Allen, D.J. Tildesley, Computer Simulation of Liquids (Oxford University Press, 1989)

60. A. Alexander-Katz, M.F. Schneider, S.W. Schneider, A. Wixforth, R.R. Netz, Phys. Rev. Lett. 97(13), 138101 (2006)

61. D. Vitkup, D. Ringe, G.A. Petsko, M. Karplus, Nat. Struct. Biol. 7, 34 (2000)

62. M. Abramowitz, I.A. Stegun, eds., Handbook of Mathematical Functions, tenth edn. (U.S. Department of Commerce, 2002) 